\title{
$T-W$ relation and free energy of the Heisenberg chain at a finite temperature
}

\author{
Pengcheng Lu, ${ }^{a, b}$ Yi Qiao, ${ }^{b, 1}$ Junpeng Cao, ${ }^{b, c, d, e, 1}$ Wen-Li Yang, ${ }^{a, e, f, 1}$ Kangjie Shi $^{a}$ \\ and Yupeng Wang ${ }^{b, e, g}$ \\ ${ }^{a}$ Institute of Modern Physics, Northwest University, \\ Xian 710127, China \\ ${ }^{b}$ Beijing National Laboratory for Condensed Matter Physics, \\ Institute of Physics, Chinese Academy of Sciences, \\ Beijing 100190, China \\ ${ }^{c}$ School of Physical Sciences, University of Chinese Academy of Sciences, \\ Beijing, China \\ ${ }^{d}$ Songshan Lake Materials Laboratory, \\ Dongguan, Guangdong 523808, China \\ ${ }^{e}$ Peng Huanwu Center for Fundamental Theory, \\ Xian 710127, China \\ ${ }^{f}$ Shaanxi Key Laboratory for Theoretical Physics Frontiers, \\ Xian 710127, China \\ ${ }^{g}$ The Yangtze River Delta Physics Research Center, \\ Liyang, Jiangsu, China \\ E-mail: lupengcheng@stumail.nwu.edu, qiaoyi_joy@foxmail.com, \\ junpengcao@iphy.ac.cn, wlyang@nwu.edu.cn, kjshi@nwu.edu.cn, \\ yupeng@iphy.ac.cn
}

Abstract: A new nonlinear integral equation (NLIE) describing the thermodynamics of the Heisenberg spin chain is derived based on the $t-W$ relation of the quantum transfer matrices. The free energy of the system in a magnetic field is thus obtained by solving the NLIE. This method can be generalized to other lattice quantum integrable models. Taking the SU(3)-invariant quantum spin chain as an example, we construct the corresponding NLIEs and compute the free energy. The present results coincide exactly with those obtained via other methods previously.

Keywords: Bethe Ansatz, Lattice Integrable Models

ArXIV EPRINT: 2105.01352

\footnotetext{
${ }^{1}$ Corresponding author.
} 


\section{Contents}

1 Introduction 1

2 Heisenberg chain and the associated QTM 3

3 T-W relation and eigenvalues of the transfer matrix 4

4 Nonlinear integral equations and the free energy 4

$\begin{array}{lll}5 & \mathrm{SU}(\boldsymbol{n}) \text {-invariant spin chain } & \mathbf{1 0}\end{array}$

5.1 T-W relations of the $\mathrm{SU}(3)$-variant chain 11

5.2 Integral representations and free energy of the periodic $\mathrm{SU}(3)$ model 16

$\begin{array}{lll}6 & \text { Conclusions } & 17\end{array}$

$\begin{array}{ll}\text { A Proof of the } t-W \text { relations (3.3) and (5.10) } & 18\end{array}$

B Numerical scheme of the spin- $\frac{1}{2}$ XXX closed chain 20

C High-temperature expansion of the Heisenberg chain 21

D Bethe ansatz solution of the SU(3) model 22

E Numerical scheme of the SU(3) model 23

F High-temperature expansion of the SU(3) model 25

\section{Introduction}

Quantum integrable systems (or exactly solvable models [1]) play important roles in investigating some non-pertubative properties of quantum field/string theory such as the planar $\mathcal{N}=4$ super-symmetric Yang-Mills (SYM) theory and the planar AdS/CFT $[2,3]$ (see also references therein). They also enhance our understanding of quantum phase transitions and critical phenomena in statistical physics [4, 5], condensed matter physics [6] and cold atom systems [7]. In the past decades, several theoretical methods $[1,8-16]$ have been developed to approach eigenvalue problem of quantum integrable models. A method to approach thermodynamic properties of quantum integrable models was first achieved by Yang and Yang for the quantum Bose gas $[17,18]$ based on the Bethe ansatz solution $[19,20]$. Later, the method (now known as thermodynamic Bethe ansatz (TBA)) was extended by Gaudin [21] and Takahashi $[22,23]$ to investigate the thermodynamics of the Heisenberg spin chain. With their methods, the free energy was finally found to be encoded by a set of infinitely many nonlinear integral equations (NLIEs). The numerical studies of these equations need some kind of truncation scheme [24-27]. An alternative approach, the so-called quantum transfer matrix (QTM) method [28-34] has also been proposed. In 
the QTM formalism, a one-dimensional quantum system at a finite temperature can be mapped into a classical system on two-dimensional inhomogeneous lattice by the TrotterSuzuki mapping [28]. The free energy of the quantum system can be expressed by the largest eigenvalue of the quantum transfer matrix and the next-largest eigenvalue provides the correlation length [34]. The advantage of QTM is that only a finite number of NLIEs are needed. Indeed, with the fusion hierarchy [28, 31, 32, 35-41] the TBA equations can be rederived. Moreover, the QTM method also allows ones to study the finite-size corrections [42-52]. Similar NLIE [53-56] exists to obtain the high-temperature expansions of the free energy up to a very high order. In addition, the transfer-matrix renormalizationgroup [54] (TMRG) has been shown to be a very powerful numerical method to study the thermodynamics of various quantum spin chain systems [57-64].

Recently, a novel $t-W$ method has been proposed to calculate physical properties of quantum integrable systems with or without $\mathrm{U}(1)$ symmetry $[65,66]$. The key point of this method lies in that a single $t-W$ relation determines the whole spectrum of the transfer matrix and the roots possess well-defined patterns. In this paper, we will construct the $t-W$ relation of the quantum transfer matrix. By analysing the root patterns of the quantum transfer matrix, a new NLIE describing the thermodynamics can be derived straightforwardly based on the $t-W$ relation.

Let us consider the Hamiltonian of the periodic Heisenberg spin chain in anti-ferromagnetic regime $(J>0)$ :

$$
H=J \sum_{n=1}^{L}\left(\sigma_{n}^{x} \sigma_{n+1}^{x}+\sigma_{n}^{y} \sigma_{n+1}^{y}+\sigma_{n}^{z} \sigma_{n+1}^{z}\right)+\frac{h}{2} \sum_{j=1}^{L} \sigma_{j}^{z},
$$

where

$$
\sigma_{1+L}^{\alpha}=\sigma_{1}^{\alpha}, \quad \text { for } \quad \alpha=x, y, z,
$$

and $\sigma^{x}, \sigma^{y}, \sigma^{z}$ are Pauli matrices. The model is one of the best studied paradigmatic models in quantum integrable systems and still remains a source of inspiration and fascinating new progress of quantum integrable systems.

The paper is organized as follows. Section 2 serves as an introduction of our notations and some basic ingredients. We also briefly review that the partition function of the Heisenberg chain at a finite temperature is expressed in the QTM formalism. In section 3, we derive the $t-W$ relation of the transfer matrix via the fusion technique. With the help of the resulting $t-W$ relation and some asymptotical behaviors of eigenvalues of the transfer matrices, we obtain the Bethe-ansatz-like equations (BAEs), which may completely determine eigenvalues. In section 4 , based on the root distributions of eigenvalues corresponding to the state with the maximus $\left|\Lambda^{(Q)}(0)\right|$, we derive a new nonlinear integral equation (NLIE) and the analytic properties, which enable us to obtain the partition function (and free energy). Taking the SU(3)-invariant spin chain as an example of the $\mathrm{SU}(n)$ case, we apply our method to obtain the corresponding free energy in section 5 . In section 6 , we summarize our results and give some discussions. Some supporting materials are given in appendices $\mathrm{A})-(\mathrm{F}$. 


\section{Heisenberg chain and the associated QTM}

The integrability of the model (1.1)-(1.2) is associated with the well-known rational sixvertex $R$-matrix

$$
R(u)=\left(\begin{array}{cccc}
u+\eta & & \\
& u & \eta & \\
& \eta & u & \\
& & & u+\eta
\end{array}\right),
$$

where $u$ is the spectral parameter and the crossing parameter $\eta=i$. The $R$-matrix satisfies the quantum Yang-Baxter equation (QYBE)

$$
R_{12}\left(u_{1}-u_{2}\right) R_{13}\left(u_{1}-u_{3}\right) R_{23}\left(u_{2}-u_{3}\right)=R_{23}\left(u_{2}-u_{3}\right) R_{13}\left(u_{1}-u_{3}\right) R_{12}\left(u_{1}-u_{2}\right),
$$

and the properties:

Initial condition : $\quad R_{12}(0)=\eta P_{12}$,

Unitarity relation : $\quad R_{12}(u) R_{21}(-u)=-\xi(u) \times$ id, $\quad \xi(u)=(u-\eta)(u+\eta)$,

Crossing relation : $\quad R_{12}(u)=V_{1} R_{12}^{t_{2}}(-u-\eta) V_{1}, \quad V=-i \sigma^{y}$,

Fusion condition : $\quad R_{12}( \pm \eta)= \pm \eta P_{12}^{( \pm)}= \pm \eta \frac{1 \pm P_{12}}{2}$,

PT-symmetry :

$R_{12}(u)=R_{21}(u)=R_{12}^{t_{1} t_{2}}(u)$,

$Z_{2}$-symmetry :

$$
\sigma_{1}^{i} \sigma_{2}^{i} R_{12}(u)=R_{12}(u) \sigma_{1}^{i} \sigma_{2}^{i}, \quad \text { for } i=x, y, z .
$$

Here $R_{21}(u)=P_{12} R_{12}(u) P_{12}$ with $P_{12}$ being the usual permutation operator and $t_{i}$ denotes transposition in the $i$-th space. Throughout this paper we adopt the standard notations: for any matrix $A \in \operatorname{End}\left(\mathbb{C}^{2}\right), A_{j}$ is an embedding operator in the tensor space $\mathbb{C}^{2} \otimes \mathbb{C}^{2} \otimes \cdots$, which acts as $A$ on the $j$-th space and as identity on the other factor spaces; $R_{i j}(u)$ is an embedding operator of R-matrix in the tensor space, which acts as identity on the factor spaces except for the $i$-th and $j$-th ones.

Let us introduce the transfer matrix $t(u)$ of the XXX closed chain [10]

$$
t^{(L)}(u)=\operatorname{tr}_{0}\left\{T_{0}^{(L)}(u)\right\}=\operatorname{tr}_{0}\left\{R_{0 L}(u) \ldots R_{01}(u)\right\},
$$

where $t r_{0}$ denotes trace over the "auxiliary space" 0 . The expression (2.1) of the $R$-matrix $R(u)$, the definition $(2.9)$ of the transfer matrix imply that

$$
t^{(L)}(u)=2 u^{L}+t^{(1)} u^{L-1}+\cdots+t^{(L-1)} u+t^{(L)} .
$$

Moreover, the Hamiltonian described by (1.1) and (1.2) can be expressed in terms of the transfer matrix $t^{(L)}(u)$ as

$$
H=H_{0}-J L+\frac{h}{2} \sum_{j=1}^{L} \sigma_{j}^{z}, \quad H_{0}=\left.2 \eta J \frac{\partial \ln t^{(L)}(u)}{\partial u}\right|_{u=0},
$$

which implies that for a small $u$ the transfer matrix has the expansion

$$
t^{(L)}(u)=t^{(L)}(0)\left(1+\frac{u}{2 \eta J} H_{0}+O\left(u^{2}\right)\right)=t^{(L)}(0) e^{\frac{u}{2 \eta J} H_{0}+O\left(u^{2}\right)},
$$


where $t^{(L)}(0)=\eta^{L} P_{1 L} \cdots P_{12}$. The above relation and the crossing-symmetry (2.5) of the $R$-matrix allow one to introduce a quantum transfer matrix $t^{(Q)}(u)[31,32]$,

$$
\begin{aligned}
t^{(Q)}(u)=\operatorname{tr}_{0}\left\{e^{\frac{h \beta}{2} \sigma_{0}^{z}}\left(R_{0 N}\left(u-\frac{2 \eta J \beta}{N}\right) R_{0 N-1}\left(u+\frac{2 \eta J \beta}{N}-\eta\right)\right) \ldots\right. \\
\left.\times R_{02}\left(u-\frac{2 \eta J \beta}{N}\right) R_{01}\left(u+\frac{2 \eta J \beta}{N}-\eta\right)\right\}
\end{aligned}
$$

where the positive real parameter $\beta$ is related to the temperature $T$ of the system as $\beta=\frac{1}{T}$. For a very large even integer $N$, the partition function $Z(\beta)$ of the spin- $\frac{1}{2} \mathrm{XXX}$ closed chain described by the Hamiltonian (1.1) and (1.2) at a temperature $T$ can be expressed in terms of the quantum transfer matrix $t^{(Q)}(u)$ by the QTM method (for details the reader is referred to ref. [34]),

$$
\begin{aligned}
Z(\beta) & =\lim _{L \rightarrow \infty} \operatorname{tr}_{1, \cdots, L}\left\{e^{-\beta H}\right\} \\
& =e^{\beta J L} \lim _{L \rightarrow \infty} \operatorname{tr}_{1, \cdots, L}\left\{\lim _{N \rightarrow \infty}\left\{\left(1-\frac{2 \beta}{N}(H+J L)+O\left(\frac{1}{N^{2}}\right)\right\}^{\frac{N}{2}}\right\}\right. \\
& =e^{\beta J L} \lim _{L \rightarrow \infty} \lim _{N \rightarrow \infty} \operatorname{tr}_{1, \cdots, N}\left\{\left\{t^{(Q)}(0)\right\}^{L}\right\} \\
& =e^{\beta J L} \lim _{L \rightarrow \infty} \lim _{N \rightarrow \infty}\left\{\Lambda^{(Q)}(0)_{\max }\right\}^{L} .
\end{aligned}
$$

Here $\Lambda^{(Q)}(0)_{\max }$ is the eigenvalue corresponding to the state with the maximus value $\left|\Lambda^{(Q)}(0)\right|$. Moreover, it was shown $[31,32,34]$ that in the limit of $N \rightarrow \infty \Lambda^{(Q)}(0)_{\max }$ is gaped from the others eigenvalues of $\Lambda^{(Q)}(0)$.

\section{T-W relation and eigenvalues of the transfer matrix}

Similarly as the quantum transfer matrix (2.11), for a large even positive integer $N$, let us introduce another transfer matrix $t(u)$

$$
t(u)=\operatorname{tr}_{0}\left\{e^{\frac{h \beta}{2} \sigma_{0}^{z}}\left(R_{0 N}\left(u-\theta_{N}\right) R_{0 N-1}\left(u-\theta_{N-1}\right) \cdots R_{02}\left(u-\theta_{2}\right) R_{01}\left(u-\theta_{1}\right)\right)\right\},
$$

where $\left\{\theta_{j} \mid j=1, \cdots, N\right\}$ are some generic complex number, which are called the inhomogeneous parameters (for the special choice of the inhomogeneous parameters, one can recover the quantum transfer matrix (2.11)). The expression (2.1) of the $R$-matrix $R(u)$, the definition (3.1) of the transfer matrix $t(u)$ imply that

$$
t(u)=2 \cosh \frac{h \beta}{2} u^{N}+t^{(1)} u^{N-1}+\cdots+t^{(N-1)} u+t^{(N)} .
$$

Moreover with the help of the fusion of $R$-matrix [70], we can derive that the transfer matrix $t(u)$ satisfies the $t-W$ relation

$$
t(u) t(u-\eta)=a(u) d(u-\eta) \times \mathrm{id}+e^{\frac{h \beta}{2}} d(u) \mathbb{W}(u),
$$


where the functions $a(u)$ and $d(u)$ are given by

$$
a(u)=e^{\frac{h \beta}{2}} \prod_{k=1}^{N}\left(u-\theta_{k}+\eta\right), \quad d(u)=e^{-\frac{h \beta}{2}} \prod_{k=1}^{N}\left(u-\theta_{k}\right),
$$

and $\mathbb{W}(u)$ (given by below (A.10)), as a function of $u$, is an operator-valued polynomial of degree $N$, which actually is some fused transfer matrix of the fundamental one. The details of the proof the $t-W$ relation (3.3) will be given in appendix A.

It is easy to shown that the transfer matrices $t(u)$ and $\mathbb{W}(u)$ commute with each other, namely,

$$
[t(u), t(v)]=[\mathbb{W}(u), \mathbb{W}(v)]=[t(u), \mathbb{W}(v)]=0,
$$

which implies that they have common eigenstates. Let $|\Psi\rangle$ be a common eigenstate of the transfer matrices with eigenvalues $\Lambda(u)$ and $W(u)$, namely,

$$
t(u)|\Psi\rangle=\Lambda(u)|\Psi\rangle, \quad \mathbb{W}(u)|\Psi\rangle=W(u)|\Psi\rangle .
$$

The operator identity (3.3) of the transfer matrices then gives rise to the corresponding relation for their eigenvalues

$$
\Lambda(u) \Lambda(u-\eta)=a(u) d(u-\eta)+e^{\frac{h \beta}{2}} d(u) W(u) .
$$

The expansion expression (3.2) and (3.7) allow us to express any eigenvalue $\Lambda(u)$ of the transfer matrix (or $W(u)$ of the fused one) in terms of its $N$ zero points $\left\{z_{j} \mid j=1, \cdots, N\right\}$ (or $\left\{w_{j} \mid j=1, \cdots, N\right\}$ ) as follow

$$
\begin{aligned}
\Lambda(u) & =2 \cosh \frac{h \beta}{2} \prod_{j=1}^{N}\left(u-z_{j}\right), \\
W(u) & =\left(4 \cosh ^{2} \frac{h \beta}{2}-1\right) \prod_{j=1}^{N}\left(u-w_{j}\right) .
\end{aligned}
$$

Taking $u$ at the $2 N$ points $\left\{z_{j} \mid j=1, \cdots, N\right\}$ and $\left\{w_{j} \mid j=1, \cdots, N\right\}$, we have the associated BAEs

$$
\begin{aligned}
a\left(z_{j}\right) d\left(z_{j}-\eta\right) & =-e^{\frac{h \beta}{2}} d\left(z_{j}\right) W\left(z_{j}\right), & & j=1, \cdots, N, \\
a\left(w_{j}\right) d\left(w_{j}-\eta\right) & =\Lambda\left(w_{j}\right) \Lambda\left(w_{j}-\eta\right), & & j=1, \cdots, N .
\end{aligned}
$$

Then $2 N$ parameters $\left\{z_{j} \mid j=1, \cdots, N\right\}$ and $\left\{w_{j} \mid j=1, \cdots, N\right\}$, which are related to the roots of the eigenvalues $\Lambda(u)$ and $W(u)$, can be determined completely by the above BAEs.

In order to investigate the thermodynamics of the spin- $\frac{1}{2}$ XXX closed chain described by the Hamiltonian (1.1) and (1.2), let us focus on the quantum transfer matrix $t^{(Q)}(u)$ given by (2.11) for a large even $N$ and denote its eigenvalue by $\Lambda^{(Q)}(u)$. In this case the inhomogeneous parameters are specially chosen by $(2.11)$ and the associated functions $a(u)$ and $d(u)$ become

$$
\begin{aligned}
& a(u)=e^{\frac{h \beta}{2}}\left\{u-\frac{2 \eta J \beta}{N}+\eta\right\}^{\frac{N}{2}}\left\{u+\frac{2 \eta J \beta}{N}\right\}^{\frac{N}{2}}, \\
& d(u)=e^{-\frac{h \beta}{2}}\left\{u-\frac{2 \eta J \beta}{N}\right\}^{\frac{N}{2}}\left\{u+\frac{2 \eta J \beta}{N}-\eta\right\}^{\frac{N}{2}}
\end{aligned}
$$


The free energy per site $f(\beta)$ is given in terms of the partition function (2.12) by

$$
\begin{aligned}
f(\beta) & =-\frac{1}{\beta} \lim _{L \rightarrow \infty} \lim _{N \rightarrow \infty} \frac{1}{L}(\ln Z(\beta)) \\
& =-J-\frac{1}{\beta} \lim _{L \rightarrow \infty} \lim _{N \rightarrow \infty}\left\{\ln \Lambda^{(Q)}(0)_{\max }\right\} .
\end{aligned}
$$

Hence it is sufficient to calculate $\Lambda^{(Q)}(u)$ of the eigenstate with $\left|\Lambda^{(Q)}(0)\right|_{\max }$. Eigenvalues of the QTM can be also obtained by the algebraic Bethe ansatz method [10] alternatively, where $\Lambda^{(Q)}(u)$ is given in terms of a homogeneous $T-Q$ relation, namely,

$$
\begin{aligned}
\Lambda^{(Q)}(u) & =a(u) \frac{Q(u-\eta)}{Q(u)}+d(u) \frac{Q(u+\eta)}{Q(u)}, \\
Q(u) & =\prod_{j=1}^{M}\left(u-\lambda_{j}\right), \quad M=0, \cdots, N,
\end{aligned}
$$

where the functions $a(u)$ and $d(u)$ are given in (3.13). The parameters $\left\{\lambda_{j} \mid j=\right.$ $1, \cdots, M ; M=0, \cdots, N\}$ satisfy the BAEs

$$
\frac{d\left(\lambda_{j}\right)}{a\left(\lambda_{j}\right)}=-\frac{Q\left(\lambda_{j}-\eta\right)}{Q\left(\lambda_{j}+\eta\right)}, \quad j=1, \cdots, M
$$

It was shown $[31,32,34]$ that the eigenvalue of the eigenstate with $\left|\Lambda^{(Q)}(0)\right|_{\max }$ belongs to the sector of $M=\frac{N}{2}$ with all the Bethe roots being real. For the simplicity, let us introduce $M=\frac{N}{2}$ in the following part of the paper, and introduce a parameter $\tau$ (a positive real number) associated with the temperature as

$$
\tau=\frac{J \beta}{M}=\frac{J}{M T}=\frac{2 J \beta}{N},
$$

and a normalized eigenvalue $\bar{\Lambda}^{(Q)}(u)$

$$
\bar{\Lambda}^{(Q)}(u)=\frac{\Lambda^{(Q)}(u)}{(u-\eta \tau+\eta)^{M}(u+\eta \tau-\eta)^{M}} .
$$

The $T-Q$ relation (3.15) allows us to express $\bar{\Lambda}^{(Q)}(u)$ as

$$
\bar{\Lambda}^{(Q)}(u)=\frac{(u+\eta \tau)^{M}}{\prod_{k=1}^{M}\left(u-\lambda_{k}\right)} \frac{\prod_{k=1}^{M}\left(u-\lambda_{k}-\eta\right)}{(u+\eta \tau-\eta)^{M}}+\frac{(u-\eta \tau)^{M}}{\prod_{k=1}^{M}\left(u-\lambda_{k}\right)} \frac{\prod_{k=1}^{M}\left(u-\lambda_{k}+\eta\right)}{(u-\eta \tau+\eta)^{M}},
$$

where the real Bethe roots satisfy the associated BAEs

$$
\frac{\left(\lambda_{j}-\eta \tau\right)^{M}\left(\lambda_{j}+\eta \tau-\eta\right)^{M}}{\left(\lambda_{j}+\eta \tau\right)^{M}\left(\lambda_{j}-\eta \tau+\eta\right)^{M}}=-\prod_{k=1}^{M} \frac{\lambda_{j}-\lambda_{k}-\eta}{\lambda_{j}-\lambda_{k}+\eta}, \quad j=1, \cdots, M
$$

The distribution of the roots of $\Lambda^{(Q)}(u)$ in figure 1 for the state with $\left|\Lambda^{(Q)}(0)\right|_{\max }$ for some small $N$ (up to 100) indicates that the corresponding $\bar{\Lambda}^{(Q)}(u)$ has the decomposition

$$
\bar{\Lambda}^{(Q)}(u)=2 \cosh \frac{h \beta}{2} \frac{\prod_{j=1}^{M}\left(u-u_{j}^{(+)}-\eta\right)\left(u-u_{j}^{(-)}+\eta\right)}{(u+\eta \tau-\eta)^{M}(u-\eta \tau+\eta)^{M}},
$$



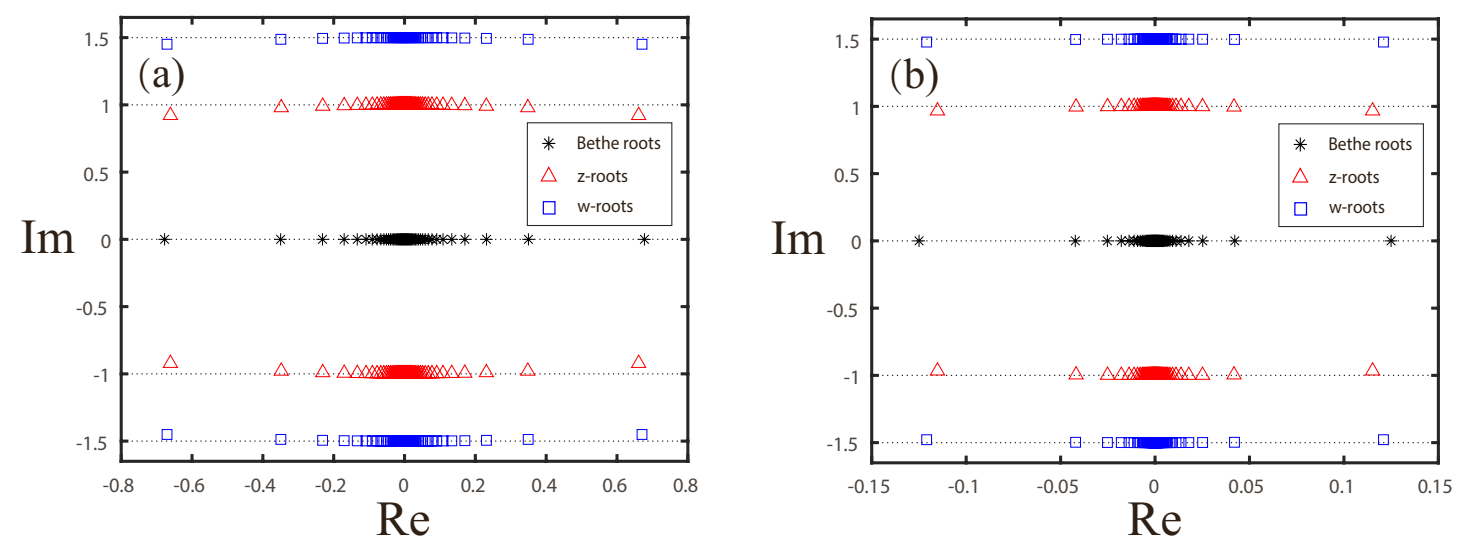

Figure 1. (Colour online) The distributions of the Bethe roots (black $*$ ), z-roots (red $\Delta$ ) and $w$-roots (blue $\square$, the roots are shifted down $\frac{\eta}{2}$ corresponding to the zeros of $W^{(Q)}\left(u+\frac{\eta}{2}\right)$ ) for the state with the maximus $\Lambda^{(Q)}(0)$ at finite temperatures $T=0.5$ (a) and $T=5$ (b). In both plots, we set $N=100$.

where the imaginary parts of $u_{j}^{( \pm)}$are close to zero for a large $N\left(\right.$ namely, $\operatorname{Im}\left(u_{j}^{( \pm)}\right) \sim 0$ ) and $\tau$ is given in (3.17). The Bethe ansatz solution (3.15) also shows that the roots of $\Lambda^{(Q)}(u)$ of the state with $\left|\Lambda^{(Q)}(0)\right|_{\max }$ indeed has the distribution (3.21). With the help of the $t-W$ relation (3.7), we can derive that the eigenvalue $W^{(Q)}(u)$ of the state with $\left|\Lambda^{(Q)}(0)\right|_{\max }$ has the decomposition

$$
W^{(Q)}(u)=\left(4 \cosh ^{2} \frac{h \beta}{2}-1\right) \prod_{j=1}^{M}\left(u-w_{j}^{(+)}-2 \eta\right)\left(u-w_{j}^{(-)}+\eta\right),
$$

where $\operatorname{Im}\left(w_{j}^{( \pm)}\right) \sim 0$ for a large $N$. Then the $t-W$ relation (3.7) for the state with the $\left|\Lambda^{(Q)}(0)\right|_{\max }$ becomes

$$
\begin{aligned}
& \bar{\Lambda}^{(Q)}\left(u+\frac{\eta}{2}\right) \bar{\Lambda}^{(Q)}\left(u-\frac{\eta}{2}\right) \\
& =4 \cosh ^{2} \frac{h \beta}{2} \frac{\prod_{j=1}^{M}\left(u-u_{j}^{(-)}+\frac{3}{2} \eta\right)\left(u-u_{j}^{(+)}-\frac{\eta}{2}\right)\left(u-u_{j}^{(-)}+\frac{\eta}{2}\right)\left(u-u_{j}^{(+)}-\frac{3}{2} \eta\right)}{\left(u-\eta \tau+\frac{3}{2} \eta\right)^{M}\left(u+\eta \tau-\frac{\eta}{2}\right)^{M}\left(u-\eta \tau+\frac{\eta}{2}\right)^{M}\left(u+\eta \tau-\frac{3}{2} \eta\right)^{M}} \\
& =\frac{\left(u+\eta \tau+\frac{\eta}{2}\right)^{M}\left(u-\eta \tau-\frac{\eta}{2}\right)^{M}}{\left(u-\eta \tau+\frac{\eta}{2}\right)^{M}\left(u+\eta \tau-\frac{\eta}{2}\right)^{M}}+\left(4 \cosh ^{2} \frac{h \beta}{2}-1\right) \frac{\prod_{j=1}^{M}\left(u-w_{j}^{(+)}-\frac{3}{2} \eta\right)\left(u-w_{j}^{(-)}+\frac{3}{2} \eta\right)}{\left(u+\eta \tau-\frac{3}{2} \eta\right)^{M}\left(u-\eta \tau+\frac{3}{2} \eta\right)^{M}} \\
& \stackrel{\text { def }}{=} q(u)+\left(4 \cosh ^{2} \frac{h \beta}{2}-1\right) \bar{w}(u)+O\left(\frac{1}{N}\right),
\end{aligned}
$$

where the functions $q(u)$ and $\bar{w}(u)$ are

$$
\begin{aligned}
q(u) & =\lim _{M \rightarrow \infty} \frac{\left(u+\eta \tau+\frac{\eta}{2}\right)^{M}\left(u-\eta \tau-\frac{\eta}{2}\right)^{M}}{\left(u-\eta \tau+\frac{\eta}{2}\right)^{M}\left(u+\eta \tau-\frac{\eta}{2}\right)^{M}}=e^{\frac{2 J \beta}{u^{2}+\frac{1}{4}}} \\
\bar{w}(u) & =\lim _{M \rightarrow \infty} \frac{\prod_{j=1}^{M}\left(u-w_{j}^{(+)}-\frac{3}{2} \eta\right)\left(u-w_{j}^{(-)}+\frac{3}{2} \eta\right)}{\left(u+\eta \tau-\frac{3}{2} \eta\right)^{M}\left(u-\eta \tau+\frac{3}{2} \eta\right)^{M}} \stackrel{\text { def }}{=} e^{-\beta \bar{\epsilon}(u)} .
\end{aligned}
$$


It is remarked that the function $\bar{\epsilon}(u)$ satisfies the analytic property:

$\bar{\epsilon}(u)$ is analytic except some singularities on the axis $\operatorname{Im}(u)= \pm \frac{3}{2}$ and $\lim _{u \rightarrow \infty} \bar{\epsilon}(u)=0$.

\section{Nonlinear integral equations and the free energy}

The decomposition (3.21) and the very $t-W$ relation (3.23) allow us to give an integral representation of $\bar{\Lambda}^{(Q)}(u)$ of the state with $\left|\Lambda^{(Q)}(0)\right|_{\max }$

$$
\begin{aligned}
\ln \bar{\Lambda}^{(Q)}(u)= & \ln 2 \cosh \frac{h \beta}{2}+\frac{1}{2 \pi i} \oint_{\mathcal{C}_{1}} d v \frac{\ln \left(\left(q(v)+\left(4 \cosh ^{2} \frac{h \beta}{2}-1\right) e^{-\beta \bar{\epsilon}(v)}\right) / 4 \cosh ^{2} \frac{h \beta}{2}\right)}{u-v-\frac{\eta}{2}} \\
& +\frac{1}{2 \pi i} \oint_{\mathcal{C}_{2}} d v \frac{\ln \left(\left(q(v)+\left(4 \cosh ^{2} \frac{h \beta}{2}-1\right) e^{-\beta \bar{\epsilon}(v)}\right) / 4 \cosh ^{2} \frac{h \beta}{2}\right)}{u-v+\frac{\eta}{2}}
\end{aligned}
$$

where the closed integral contour $\mathcal{C}_{1}$ is surrounding the axis of $\operatorname{Im}(v)=\frac{1}{2}$, while $\mathcal{C}_{2}$ is surrounding the axis of $\operatorname{Im}(v)=-\frac{1}{2}$. With the help of the $t-W$ relation (3.23) and the integral representation (4.1), we can derive a NLIE of the function $\bar{\epsilon}(u)$

$$
\begin{aligned}
& \ln \left(q(u)+\left(4 \cosh ^{2} \frac{h \beta}{2}-1\right) e^{-\beta \bar{\epsilon}(u)}\right)=2 \ln 2 \cosh \frac{h \beta}{2} \\
& \quad+\frac{1}{2 \pi i} \oint_{\mathcal{C}_{1}} d v\left(\frac{1}{u-v}+\frac{1}{u-v-\eta}\right) \ln \left(\left(q(v)+\left(4 \cosh ^{2} \frac{h \beta}{2}-1\right) e^{-\beta \bar{\epsilon}(v)}\right) / 4 \cosh ^{2} \frac{h \beta}{2}\right) \\
& \quad+\frac{1}{2 \pi i} \oint_{\mathcal{C}_{2}} d v\left(\frac{1}{u-v+\eta}+\frac{1}{u-v}\right) \ln \left(\left(q(v)+\left(4 \cosh ^{2} \frac{h \beta}{2}-1\right) e^{-\beta \bar{\epsilon}(v)}\right) / 4 \cosh ^{2} \frac{h \beta}{2}\right) .
\end{aligned}
$$

Due to the fact that the roots and the poles of $\bar{\Lambda}^{(Q)}(u)$ locate nearly on the two lines with imaginary parts close to \pm 1 (see the decomposition (3.21)), we can use the Fourier transformation to obtain another integral representation of $\bar{\Lambda}^{(Q)}(u)$

$$
\begin{aligned}
\ln \bar{\Lambda}^{(Q)}(u) & =\int_{-\infty}^{+\infty} \frac{d v}{2 \cosh \pi(u-v)}\left\{\ln \bar{\Lambda}^{(Q)}\left(v+\frac{\eta}{2}\right)+\ln \bar{\Lambda}^{(Q)}\left(v-\frac{\eta}{2}\right)\right\} \\
& =\int_{-\infty}^{+\infty} \frac{d v}{2 \cosh \pi(u-v)}\left\{\frac{2 J \beta}{v^{2}+\frac{1}{4}}+\ln \left(1+\left(4 \cosh ^{2} \frac{h \beta}{2}-1\right) q^{-1}(v) \bar{w}(v)\right)\right\}
\end{aligned}
$$

where we have used $\eta=i$. Let us introduce the dressing energy function $\epsilon(u)$

$$
\epsilon(u)=-\frac{1}{\beta} \ln \left(q^{-1}(u) \bar{w}(u)\right)=\frac{2 J}{u^{2}+\frac{1}{4}}+\bar{\epsilon}(u), \quad \lim _{u \rightarrow \infty} \epsilon(u)=0 .
$$

It is believed that the analytic property (3.26) and the NLIE (4.2) and the asymptotical behavior (4.4) might completely determine the function $\bar{\epsilon}(u)$. 


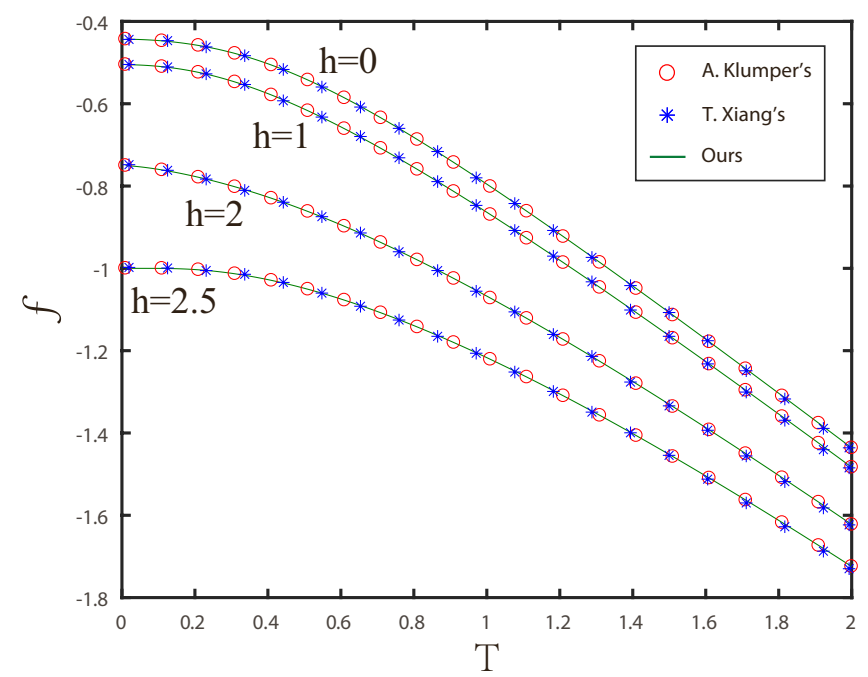

Figure 2. Free energy $f$ vs $T$ for the closed XXX chain in different magnetic fields.

Finally we obtain the free energy of the XXX chain described by the Hamiltonian (1.1)$(1.2)$ as

$$
\begin{aligned}
f(\beta) & =J-\frac{1}{\beta} \ln \bar{\Lambda}^{(Q)}(0) \\
& =J-J \int_{-\infty}^{+\infty} \frac{d v}{\cosh \pi v} \frac{1}{v^{2}+\frac{1}{4}}-\frac{1}{\beta} \int_{-\infty}^{+\infty} \frac{d v}{2 \cosh \pi v} \ln \left(1+\left(4 \cosh ^{2} \frac{h \beta}{2}-1\right) e^{-\beta \epsilon(v)}\right) \\
& =e_{g}-\frac{1}{\beta} \int_{-\infty}^{+\infty} \frac{d v}{2 \cosh \pi v} \ln \left(1+\left(4 \cosh ^{2} \frac{h \beta}{2}-1\right) e^{-\beta \epsilon(v)}\right),
\end{aligned}
$$

where $e_{g}=J-J \int_{-\infty}^{+\infty} \frac{d v}{\cosh \pi v} \frac{1}{v^{2}+\frac{1}{4}}$ is the energy of the ground state for the XXX chain (1.1)(1.2) [23] and the dressing energy $\epsilon(u)$ satisfying the relations (3.26), (4.2) and (4.4).

Using the numerical iterative procedure in appendix B, we obtain the free energy $f$ variation with temperature $T$ in different magnetic fields as shown in figure 2. From the figure, we find that our result coincides well with the those of [31, 32] and [60] obtained with different approaches. Moreover, the analytic property (3.26) and the NLIE (4.2) allow us to give the HTE of the free energy as

$$
f / T=-\ln (2 \cosh (h / T))-\frac{J}{T} \tanh ^{2}(h / T)-\frac{3 J^{2}}{2 T^{2}}\left(1-\tanh ^{4}(h / T)\right)+\cdots,
$$

which recovers that of [56] obtained previously with a different approach. The details of the derivation of (4.6) will be given in appendix C.

Our method to obtain the NLIEs is more straightforward and easily extensible to other quantum integrable spin chains associated other Lie algebras. We will apply the method to construct the corresponding NLIEs for the $\mathrm{SU}(n)$ quantum spin chain in the next section. Moreover, the procedure to obtain the NLIEs does not directly depend on the Bethe ansatz solution (3.15) and (3.20) of the model, which is related only to the patterns of the root distributions of $\Lambda^{Q}(u)$ and the fused ones. The roots distributions can be obtained by 
directly solving the equations (3.10)-(3.11) (or the below equations (5.24)-(5.27) for the $\mathrm{SU}(3)$-case).

\section{$5 \quad \mathrm{SU}(n)$-invariant spin chain}

Let $\mathbf{V}$ denote an $n$-dimensional linear space. The Hamiltonian of SU(n)-invariant quantum spin system on a $L$-sites lattice with the periodic boundary condition is given by [10]

$$
H=J \sum_{j=1}^{L} P_{j j+1}, \quad P_{j j+1}=\sum_{\mu, \nu=1}^{n} E_{j}^{\mu, \nu} E_{j+1}^{\nu, \mu},
$$

where $P_{j j+1}$ is permutation operator and $\left(E^{\mu, \nu}\right)_{\beta}^{\alpha}=\delta_{\alpha}^{\mu} \delta_{\nu}^{\beta}$ with $\alpha, \beta, \gamma, \delta=1, \cdots, n$. The integrability of the system (5.1) is guaranteed by the $\mathrm{SU}(n)$-invariant $R$-matrix $R(u) \in$ $\operatorname{End}(\mathbf{V} \otimes \mathbf{V})[67,68]$.

$$
R_{12}(u)=u+\eta P_{12} .
$$

Besides the QYBE, the $R$-matrix satisfies the properties:

Initial condition : $\quad R_{12}(0)=\eta P_{12}$,

Unitarity relation: $\quad R_{12}(u) R_{21}(-u)=-\rho_{1}(u) \times \mathrm{id}, \quad \rho_{1}(u)=(u-\eta)(u+\eta)$,

Crossing-unitarity : $\quad R_{12}^{t_{1}}(u) R_{21}^{t_{1}}(-u-n \eta)=-\rho_{2}(u) \times \mathrm{id}, \quad \rho_{2}(u)=u(u+3 \eta)$,

PT-symmetry: $\quad R_{12}(u)=R_{21}(u)=R_{12}^{t_{1} t_{2}}(u)$,

Fusion conditions : $\quad R_{12}(-\eta)=-2 \eta P_{12}^{(-)}, \quad R_{12}(\eta)=2 \eta P_{12}^{(+)}$.

The corresponding QTM can be constructed as follow [52]

$$
t_{1}^{(Q)}(u)=\operatorname{tr}_{0}\left\{e^{\frac{h \beta}{2} S_{0}}\left(R_{0 N}(u-\eta \tau) R_{N-10}^{t_{N-1}}(u+\eta \tau)\right) \cdots\left(R_{02}(u-\eta \tau) R_{10}^{t_{1}}(u+\eta \tau)\right)\right\}
$$

where the diagonal matrix $S_{0}=\operatorname{diag}\left(\mu_{1}, \mu_{2}, \ldots, \mu_{n}\right)$ is related to the external field and $\tau$ is given in (3.17). In the case of the $\mathrm{SU}(3)$ invariant spin chain, we have $S_{0}=S_{0}^{z}=$ $\operatorname{diag}(1,0,-1)$. The expression (5.2) of the $R$-matrix $R(u)$, the definition (5.8) of the QTM imply that

$$
t_{1}^{(Q)}(u)=\left(\sum_{j=1}^{n} e^{\frac{h \beta}{2} \mu_{j}}\right) u^{N}+t_{1}^{(1)} u^{N-1}+\cdots+t_{1}^{(N-1)} u+t_{1}^{(N)} .
$$

With the help of the fusion $[69,70]$ of the $R$-matrix we can introduce some fused quantum transfer matrices ${ }^{1}\left\{t_{i}^{(Q)}(u) \mid i=2, \cdots, n-1\right\}$ which are related to the representations associated with the other $n-2$ fundamental highest weights of $s u(n)$ algebra and their counterparts $\left\{\mathbb{W}_{i}^{(Q)}(u) \mid i=1, \cdots, n-1\right\}$. Using the method developed in [71] we can derive that the fused transfer matrices satisfy the associated $t-W$ relations

$$
t_{m}^{(Q)}(u) t_{m}^{(Q)}(u-\eta)=t_{m-1}^{(Q)}(u-\eta) t_{m+1}^{(Q)}(u)+a_{m}(u) \mathbb{W}_{m}^{(Q)}(u), \quad m=1, \cdots, n-1 .
$$

\footnotetext{
${ }^{1}$ It is remarked that the fused transfer matrices $\left\{t_{i}^{(Q)}(u) \mid i=2, \cdots, n-1\right\}$ in this paper correspond to those $\left\{\tau_{i}^{(p)}(u) \mid i=2, \cdots, n-1\right\}$ in [71].
} 
The operators $t_{0}^{(Q)}(u), t_{n}^{(Q)}(u)$ and the functions $a_{m}(u)$ are given by

$$
\begin{aligned}
t_{0}^{(Q)}(u) & =[(u-\eta \tau-\eta)(u+\eta \tau)]^{\frac{N}{2}} \times \mathrm{id}, & t_{n}^{(Q)}(u) & =[(u+\eta \tau-n \eta)(u-\eta \tau+\eta)]^{\frac{N}{2}} \times \mathrm{id}, \\
a_{m}(u) & =[(u+\eta \tau-m \eta)(u-\eta \tau)]^{\frac{N}{2}}, & m & =1, \cdots, n-1 .
\end{aligned}
$$

The proof of the relations (5.10) will be given in appendix A.

Some remarks are in order. We have introduced the $2 n-3$ extra (or auxiliary) fused transfer matrices $\left\{t_{i}^{(Q)}(u) \mid i=2, \cdots, n-1\right\}$ and $\left\{\mathbb{W}_{i}^{(Q)}(u) \mid i=1, \cdots, n-1\right\}$. Hence in order to determine the eigenvalue $\Lambda^{(Q)}(u)$ of the original quantum transfer matrix $t^{(Q)}(u)$, we need to further introduce $2 n-3$ auxiliary functions (cf., $2^{n}-2$ auxiliary functions for the $\mathrm{SU}(n)$ case $[52,72])$ which correspond to the eigenvalues of the resulting fused transfer matrices.

\subsection{T-W relations of the $\mathrm{SU}(3)$-variant chain}

Taking the SU(3)-invariant spin chain as an example, we shall show how our method works in the following parts of the section. The Hamiltonian of the SU(3)-invariant closed spin chain is given by

$$
H=J \sum_{j=1}^{L} P_{j, j+1}, \quad P_{j, j+1}=\sum_{\mu, \nu=1}^{3} E_{j}^{\mu, \nu} E_{j+1}^{\nu, \mu}
$$

with the periodic boundary condition

$$
E_{L+1}^{\mu, \nu}=E_{1}^{\mu, \nu}, \quad \text { for } \quad \mu, \nu=1,2,3 .
$$

The associated $R$-matrix reads

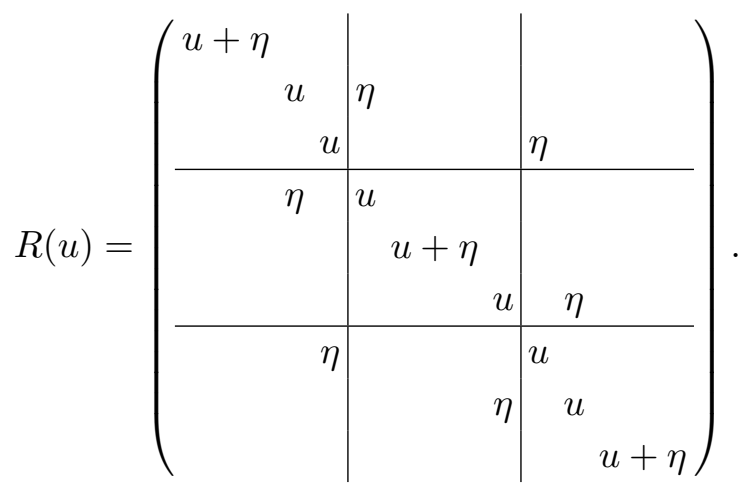

For the periodic SU(3) model with an external field $h$, the corresponding QTM can be constructed as follow [52]

$$
t_{1}^{(Q)}(u)=\operatorname{tr}_{0}\left\{e^{\frac{h \beta}{2} S_{0}^{z}}\left(R_{0 N}(u-\eta \tau) R_{N-10}^{t_{N-1}}(u+\eta \tau)\right) \cdots\left(R_{02}(u-\eta \tau) R_{10}^{t_{1}}(u+\eta \tau)\right)\right\}
$$

where the operator $S_{0}^{z}=\operatorname{diag}(1,0,-1)$. The expression (5.15) of the $R$-matrix $R(u)$, the definition (5.16) of the QTM imply that

$$
t_{1}^{(Q)}(u)=\left(2 \cosh \frac{h \beta}{2}+1\right) u^{N}+t_{1}^{(1)} u^{N-1}+\cdots+t_{1}^{(N-1)} u+t_{1}^{(N)} .
$$


The corresponding $t-W$ relations $^{2}$ (5.10) read

$$
\begin{aligned}
t_{1}^{(Q)}(u) t_{1}^{(Q)}(u-\eta)= & {[(u+\eta \tau)(u-\eta \tau-\eta)]^{\frac{N}{2}} t_{2}^{(Q)}\left(u-\frac{\eta}{2}\right) } \\
& +[(u+\eta \tau-\eta)(u-\eta \tau)]^{\frac{N}{2}} \mathbb{W}_{1}^{(Q)}(u) \\
t_{2}^{(Q)}(u) t_{2}^{(Q)}(u-\eta)= & {\left[\left(u+\eta \tau-\frac{5}{2} \eta\right)\left(u-\eta \tau+\frac{3}{2} \eta\right)\right]^{\frac{N}{2}} t_{1}^{(Q)}\left(u-\frac{\eta}{2}\right) } \\
& +\left[\left(u+\eta \tau-\frac{3}{2} \eta\right)\left(u-\eta \tau+\frac{\eta}{2}\right)\right]^{\frac{N}{2}} \mathbb{W}_{2}^{(Q)}(u) .
\end{aligned}
$$

The resulting transfer matrices $t_{2}^{(Q)}(u), \mathbb{W}_{1}^{(Q)}(u)$ and $\mathbb{W}_{2}^{(Q)}(u)$, as the functions of $u$, are three operator-valued polynomials of degree $N$. In addition, the transfer matrices $t_{1}^{(Q)}(u)$, $t_{2}^{(Q)}(u), \mathbb{W}_{1}^{(Q)}(u)$ and $\mathbb{W}_{2}^{(Q)}(u)$ commute with each other,

$$
\left[t_{i}^{(Q)}(u), t_{j}^{(Q)}(v)\right]=\left[\mathbb{W}_{i}^{(Q)}(u), \mathbb{W}_{j}^{(Q)}(v)\right]=\left[t_{i}^{(Q)}(u), \mathbb{W}_{j}^{(Q)}(v)\right]=0, \quad i, j=1,2
$$

The commutativity $(5.20)$ of the transfer matrices $t_{1}^{(Q)}(u), t_{2}^{(Q)}(u), \mathbb{W}_{1}^{(Q)}(u)$ and $\mathbb{W}_{2}^{(Q)}(u)$ with different spectral parameters implies that they have common eigenstates. Let $|\Psi\rangle$ be a common eigenstate of the QTMs with the eigenvalues $\Lambda_{1}^{(Q)}(u), \Lambda_{2}^{(Q)}(u), W_{1}^{(Q)}(u)$ and $W_{2}^{(Q)}(u)$, namely

$$
t_{i}^{(Q)}(u)|\Psi\rangle=\Lambda_{i}^{(Q)}(u)|\Psi\rangle, \quad \mathbb{W}_{i}^{(Q)}(u)|\Psi\rangle=W_{i}^{(Q)}(u)|\Psi\rangle, \quad i=1,2 .
$$

The operator identities (5.18) and (5.19) of the QTMs then give rise to the corresponding relations for their eigenvalues

$$
\begin{aligned}
\Lambda_{1}^{(Q)}(u) \Lambda_{1}^{(Q)}(u-\eta)= & {[(u+\eta \tau)(u-\eta \tau-\eta)]^{\frac{N}{2}} \Lambda_{2}^{(Q)}\left(u-\frac{\eta}{2}\right) } \\
& +[(u+\eta \tau-\eta)(u-\eta \tau)]^{\frac{N}{2}} W_{1}^{(Q)}(u), \\
\Lambda_{2}^{(Q)}(u) \Lambda_{2}^{(Q)}(u-\eta)= & {\left[\left(u+\eta \tau-\frac{5}{2} \eta\right)\left(u-\eta \tau+\frac{3}{2} \eta\right)\right]^{\frac{N}{2}} \Lambda_{1}^{(Q)}\left(u-\frac{\eta}{2}\right) } \\
& +\left[\left(u+\eta \tau-\frac{3}{2} \eta\right)\left(u-\eta \tau+\frac{\eta}{2}\right)\right]^{\frac{N}{2}} W_{2}^{(Q)}(u) .
\end{aligned}
$$

The expansion expression (5.17), (5.21) and (5.22) allow us to express any eigenvalue $\Lambda_{1}^{(Q)}(u)$ (or $\Lambda_{2}^{(Q)}(u), W_{1}^{(Q)}(u)$ and $\left.W_{2}^{(Q)}(u)\right)$ of the QTM in terms of its $N$ zero points $\left\{z_{j}^{(1)} \mid j=1, \cdots, N\right\}$ (or $\left\{z_{j}^{(2)} \mid j=1, \cdots, N\right\},\left\{w_{j}^{(1)} \mid j=1, \cdots, N\right\}$ and $\left\{w_{j}^{(2)} \mid j=1, \cdots, N\right\}$ ) as follow

$$
\Lambda_{i}^{(Q)}(u)=b(\beta) \prod_{j=1}^{N}\left(u-z_{j}^{(i)}\right), \quad W_{i}^{(Q)}(u)=\left(b^{2}(\beta)-b(\beta)\right) \prod_{j=1}^{N}\left(u-w_{j}^{(i)}\right), \quad i=1,2,
$$

\footnotetext{
${ }^{2}$ For later calculative and notational convenience, we shift the spectral parameter $u$ of the transfer matrix $t_{2}^{(Q)}(u)$ to be $u-\frac{\eta}{2}$ in the first $t-W$ relation (5.18).
} 
where $b(\beta)=2 \cosh \frac{h \beta}{2}+1$. Taking $u$ at the $4 N$ points $\left\{z_{j}^{(i)} \mid j=1, \cdots, N\right\}$ and $\left\{w_{j}^{(i)} \mid j=\right.$ $1, \cdots, N\}(i=1,2)$, we have the associated BAEs

$$
\begin{aligned}
& {\left[\left(z_{j}^{(1)}+\eta \tau\right)\left(z_{j}^{(1)}-\eta \tau-\eta\right)\right]^{\frac{N}{2}} \Lambda_{2}^{(Q)}\left(z_{j}^{(1)}-\frac{\eta}{2}\right)} \\
& \quad=-\left[\left(z_{j}^{(1)}+\eta \tau-\eta\right)\left(z_{j}^{(1)}-\eta \tau\right)\right]^{\frac{N}{2}} W_{1}^{(Q)}\left(z_{j}^{(1)}\right), \quad j=1, \cdots, N \\
& \quad\left[\left(w_{j}^{(1)}+\eta \tau\right)\left(w_{j}^{(1)}-\eta \tau-\eta\right)\right]^{\frac{N}{2}} \Lambda_{2}^{(Q)}\left(w_{j}^{(1)}-\frac{\eta}{2}\right) \\
& \quad=\Lambda_{1}^{(Q)}\left(w_{j}^{(1)}\right) \Lambda_{1}^{(Q)}\left(w_{j}^{(1)}-\eta\right), \quad j=1, \cdots, N \\
& {\left[\left(z_{j}^{(2)}+\eta \tau-\frac{5}{2} \eta\right)\left(z_{j}^{(2)}-\eta \tau+\frac{3}{2} \eta\right)\right]^{\frac{N}{2}} \Lambda_{1}^{(Q)}\left(z_{j}^{(2)}-\frac{\eta}{2}\right)} \\
& \quad=-\left[\left(z_{j}^{(2)}+\eta \tau-\frac{3}{2} \eta\right)\left(z_{j}^{(2)}-\eta \tau+\frac{\eta}{2}\right)\right]^{\frac{N}{2}} W_{2}^{(Q)}\left(z_{j}^{(2)}\right), \quad j=1, \cdots, N, \\
& \quad\left[\left(w_{j}^{(2)}+\eta \tau-\frac{5}{2} \eta\right)\left(w_{j}^{(2)}-\eta \tau+\frac{3}{2} \eta\right)\right]^{\frac{N}{2}} \Lambda_{1}^{(Q)}\left(w_{j}^{(2)}-\frac{\eta}{2}\right) \\
& \quad=\Lambda_{2}^{(Q)}\left(w_{j}^{(2)}\right) \Lambda_{2}^{(Q)}\left(w_{j}^{(2)}-\eta\right), \quad j=1, \cdots, N .
\end{aligned}
$$

Then $4 N$ parameters $\left\{z_{j}^{(i)} \mid j=1, \cdots, N\right\}$ and $\left\{w_{j}^{(i)} \mid j=1, \cdots, N\right\}(i=1,2)$, which are related to the roots of the eigenvalues $\left\{\Lambda_{i}^{(Q)}(u), W_{i}^{(Q)}(u) \mid i=1,2\right\}$, can be determined completely by the above BAEs.

Similarly, the normalized eigenvalues $\bar{\Lambda}_{1}^{(Q)}(u)$ and $\bar{\Lambda}_{2}^{(Q)}(u)$ are defined

$$
\begin{aligned}
& \bar{\Lambda}_{1}^{(Q)}(u)=\frac{\Lambda_{1}^{(Q)}(u)}{(u-\eta \tau+\eta)^{M}(u+\eta \tau-\eta)^{M}}, \\
& \bar{\Lambda}_{2}^{(Q)}(u)=\frac{\Lambda_{2}^{(Q)}(u)}{\left(u-\eta \tau+\frac{3}{2} \eta\right)^{M}\left(u+\eta \tau-\frac{3}{2} \eta\right)^{M}} .
\end{aligned}
$$

Numerical study with some small $N$ (up to 12) for the distributions in figure 3 of the roots of $\left\{\Lambda_{i}^{(Q)}(u) \mid i=1,2\right\}$ for the state with $\left|\Lambda_{1}^{(Q)}(0)\right|_{\max }$ indicts that the corresponding $\left\{\bar{\Lambda}_{i}^{(Q)}(u) \mid i=1,2\right\}$ have the decompositions

$$
\begin{aligned}
& \bar{\Lambda}_{1}^{(Q)}(u)=b(\beta) \frac{\prod_{j=1}^{M}\left(u-u_{1, j}^{(+)}-\eta\right)\left(u-u_{1, j}^{(-)}+\eta\right)}{(u+\eta \tau-\eta)^{M}(u-\eta \tau+\eta)^{M}} \\
& \bar{\Lambda}_{2}^{(Q)}(u)=b(\beta) \frac{\prod_{j=1}^{M}\left(u-u_{2, j}^{(+)}-\frac{3}{2} \eta\right)\left(u-u_{2, j}^{(-)}+\frac{3}{2} \eta\right)}{\left(u+\eta \tau-\frac{3}{2} \eta\right)^{M}\left(u-\eta \tau+\frac{3}{2} \eta\right)^{M}}
\end{aligned}
$$

where $\left\{\operatorname{Im}\left(u_{i, j}^{( \pm)}\right) \sim 0 \mid i=1,2\right\}$ for a large $N$. The Bethe ansatz solutions (given in appendix D, see the $T-Q$ relations below (D.4) and (D.6)) indeed confirm that the roots of $\left\{\Lambda_{i}^{(Q)}(u) \mid i=1,2\right\}$ for the state with $\left|\Lambda^{(Q)}(0)\right|_{\max }$ do have the distributions (5.30) and (5.31). With the help of the $t-W$ relations (5.21)-(5.22) and numerical results as shown in figure 4 , we can derive that the eigenvalues $\left\{W_{i}^{(Q)}(u) \mid i=1,2\right\}$ for the state with $\left|\Lambda_{1}^{(Q)}(0)\right|_{\max }$ have 

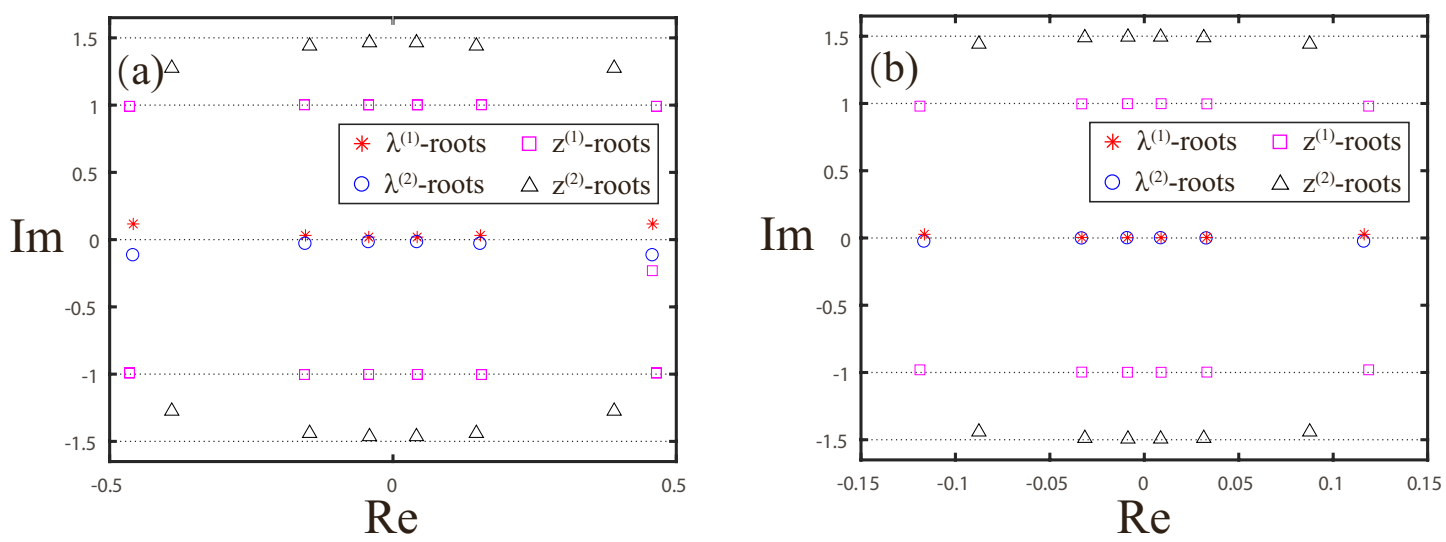

Figure 3. (Colour online) The distributions of the $\lambda^{(1)}$-roots (red $*$ ), $\lambda^{(2)}$-roots (blue $\left.\circ\right), z^{(1)}$-roots (mauve $\square$ ) and $z^{(2)}$-roots (black $\triangle$ ) for the state with the maximus $\Lambda^{(Q)}(0)$ at finite temperatures $T=1$ (a) and $T=5$ (b). In both plots, we set $N=12$.
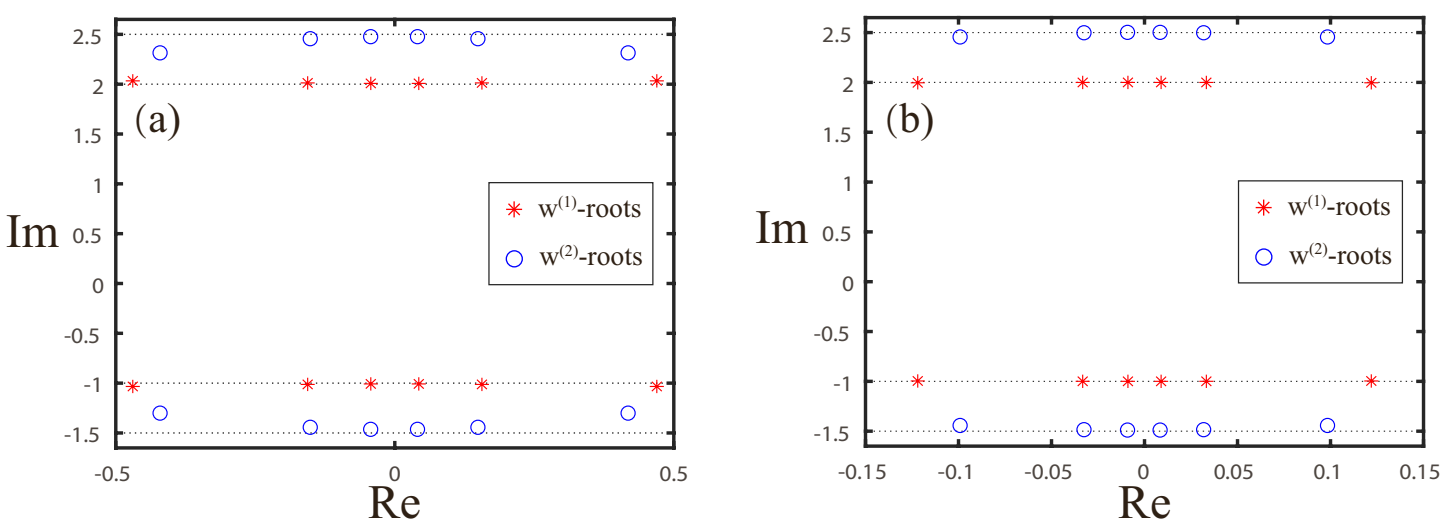

Figure 4. (Colour online) The distributions of the $\omega^{(1)}$-roots (red $*$ ), and $\omega^{(2)}$-roots (blue $\circ$ ) for the state with the maximus $\Lambda^{(Q)}(0)$ at finite temperatures $T=1$ (a) and $T=5$ (b). In both plots, we set $N=12$.

the decompositions

$$
\begin{aligned}
& W_{1}^{(Q)}(u)=\left(b^{2}(\beta)-b(\beta)\right) \prod_{j=1}^{M}\left(u-w_{1, j}^{(+)}-2 \eta\right)\left(u-w_{1, j}^{(-)}+\eta\right), \\
& W_{2}^{(Q)}(u)=\left(b^{2}(\beta)-b(\beta)\right) \prod_{j=1}^{M}\left(u-w_{2, j}^{(+)}-\frac{5}{2} \eta\right)\left(u-w_{2, j}^{(-)}+\frac{3}{2} \eta\right),
\end{aligned}
$$

where $\left\{\operatorname{Im}\left(w_{i, j}^{( \pm)}\right) \sim 0 \mid i=1,2\right\}$ for a large $N$. Then the $t-W$ relations (5.21) and (5.22) for the state with $\left|\Lambda_{1}^{(Q)}(0)\right|_{\max }$ become

$$
\begin{aligned}
& \bar{\Lambda}_{1}^{(Q)}\left(u+\frac{\eta}{2}\right) \bar{\Lambda}_{1}^{(Q)}\left(u-\frac{\eta}{2}\right) \\
& \quad=b^{2}(\beta) \frac{\prod_{j=1}^{M}\left(u-u_{1, j}^{(-)}+\frac{3}{2} \eta\right)\left(u-u_{1, j}^{(+)}-\frac{\eta}{2}\right)\left(u-u_{1, j}^{(-)}+\frac{\eta}{2}\right)\left(u-u_{1, j}^{(+)}-\frac{3}{2} \eta\right)}{\left(u-\eta \tau+\frac{3}{2} \eta\right)^{M}\left(u+\eta \tau-\frac{\eta}{2}\right)^{M}\left(u-\eta \tau+\frac{\eta}{2}\right)^{M}\left(u+\eta \tau-\frac{3}{2} \eta\right)^{M}}
\end{aligned}
$$




$$
\begin{aligned}
&=b(\beta) \frac{\left(u+\eta \tau+\frac{\eta}{2}\right)^{M}\left(u-\eta \tau-\frac{\eta}{2}\right)^{M} \prod_{j=1}^{M}\left(u-u_{2, j}^{(+)}-\frac{3}{2} \eta\right)\left(u-u_{2, j}^{(-)}+\frac{3}{2} \eta\right)}{\left(u-\eta \tau+\frac{\eta}{2}\right)^{M}\left(u+\eta \tau-\frac{\eta}{2}\right)^{M}} \\
&+\left(b^{2}(\beta)-b(\beta)\right) \frac{\prod_{j=1}^{M}\left(u-w_{1, j}^{(+)}-\frac{3}{2} \eta\right)\left(u-w_{1, j}^{(-)}+\frac{3}{2} \eta\right)}{\left(u+\eta \tau-\frac{3}{2} \eta\right)^{M}\left(u-\eta \tau+\frac{3}{2} \eta\right)^{M}} \\
&=b(\beta) q(u) \bar{\lambda}_{2}(u)+\left(b^{2}(\beta)-b(\beta)\right) \bar{w}_{1}(u)+O\left(\frac{1}{N}\right), \\
& \bar{\Lambda}_{2}^{(Q)}\left(u+\frac{\eta}{2}\right) \bar{\Lambda}_{2}^{(Q)}\left(u-\frac{\eta}{2}\right) \\
&=b^{2}(\beta) \frac{\prod_{j=1}^{M}\left(u-u_{2, j}^{(-)}+2 \eta\right)\left(u-u_{2, j}^{(+)}-\eta\right)\left(u-u_{2, j}^{(-)}+\eta\right)\left(u-u_{2, j}^{(+)}-2 \eta\right)}{(u-\eta \tau+2 \eta)^{M}(u+\eta \tau-\eta)^{M}(u-\eta \tau+\eta)^{M}(u+\eta \tau-2 \eta)^{M}} \\
&=b(\beta) \frac{\prod_{j=1}^{M}\left(u-u_{1, j}^{(+)}-\eta\right)\left(u-u_{1, j}^{(-)}+\eta\right)}{(u+\eta \tau-\eta)^{M}(u-\eta \tau+\eta)^{M}} \\
& \quad+\left(b^{2}(\beta)-b(\beta)\right) \frac{\prod_{j=1}^{M}\left(u-w_{2, j}^{(+)}-2 \eta\right)\left(u-w_{2, j}^{(-)}+2 \eta\right)}{(u+\eta \tau-2 \eta)^{M}(u-\eta \tau+2 \eta)^{M}} \\
&=b(\beta) \bar{\lambda}_{1}(u)+\left(b^{2}(\beta)-b(\beta)\right) \bar{w}_{2}(u)+O\left(\frac{1}{N}\right),
\end{aligned}
$$

where the functions $\bar{\lambda}_{1}(u), \bar{\lambda}_{2}(u), \bar{w}_{1}(u)$ and $\bar{w}_{2}(u)$ are

$$
\begin{aligned}
& \bar{\lambda}_{1}(u)=\lim _{M \rightarrow \infty} \frac{\prod_{j=1}^{M}\left(u-u_{1, j}^{(+)}-\eta\right)\left(u-u_{1, j}^{(-)}+\eta\right)}{(u+\eta \tau-\eta)^{M}(u-\eta \tau+\eta)^{M}} \stackrel{\text { def }}{=} e^{-\beta \bar{\varepsilon}_{1}(u)}, \\
& \bar{\lambda}_{2}(u)=\lim _{M \rightarrow \infty} \frac{\prod_{j=1}^{M}\left(u-u_{2, j}^{(+)}-\frac{3}{2} \eta\right)\left(u-u_{2, j}^{(-)}+\frac{3}{2} \eta\right)}{\left(u+\eta \tau-\frac{3}{2} \eta\right)^{M}\left(u-\eta \tau+\frac{3}{2} \eta\right)^{M}} \stackrel{\text { def }}{=} e^{-\beta \bar{\varepsilon}_{2}(u)}, \\
& \bar{w}_{1}(u)=\lim _{M \rightarrow \infty} \frac{\prod_{j=1}^{M}\left(u-w_{1, j}^{(+)}-\frac{3}{2} \eta\right)\left(u-w_{1, j}^{(-)}+\frac{3}{2} \eta\right)}{\left(u+\eta \tau-\frac{3}{2} \eta\right)^{M}\left(u-\eta \tau+\frac{3}{2} \eta\right)^{M}} \stackrel{\text { def }}{=} e^{-\beta \bar{\varepsilon}_{3}(u)}, \\
& \bar{w}_{2}(u)=\lim _{M \rightarrow \infty} \frac{\prod_{j=1}^{M}\left(u-w_{2, j}^{(+)}-2 \eta\right)\left(u-w_{2, j}^{(-)}+2 \eta\right)}{(u+\eta \tau-2 \eta)^{M}(u-\eta \tau+2 \eta)^{M}} \stackrel{\text { def }}{=} e^{-\beta \bar{\varepsilon}_{4}(u)} .
\end{aligned}
$$

It is remarked that the functions $\left\{\bar{\varepsilon}_{i}(u) \mid i=1, \ldots, 4\right\}$ satisfy the analytic properties:

$\bar{\varepsilon}_{1}(u)$ is analytic except some singularities on the axis $\operatorname{Im}(u)= \pm 1$, $\bar{\varepsilon}_{2}(u)$ and $\bar{\varepsilon}_{3}(u)$ are analytic except some singularities on the axis $\operatorname{Im}(u)= \pm \frac{3}{2}$, $\bar{\varepsilon}_{4}(u)$ is analytic except some singularities on the axis $\operatorname{Im}(u)= \pm 2$, $\lim _{u \rightarrow \infty} \bar{\varepsilon}_{i}(u)=0, \quad i=1, \ldots, 4$. 


\subsection{Integral representations and free energy of the periodic $\mathrm{SU}(3)$ model}

The decompositions (5.30)-(5.31) and the very $t-W$ relations (5.34)-(5.35) allow us to give the integral representations of $\bar{\Lambda}_{1}^{(Q)}(u)$ and $\bar{\Lambda}_{2}^{(Q)}(u)$

$$
\begin{aligned}
\ln \bar{\Lambda}_{1}^{(Q)}(u)= & \ln b(\beta)+\frac{1}{2 \pi i} \oint_{\mathcal{C}_{1}^{\prime}} d v \frac{\ln \left(\left(q(v) e^{-\beta \bar{\varepsilon}_{2}(v)}+(b(\beta)-1) e^{-\beta \bar{\varepsilon}_{3}(v)}\right) / b(\beta)\right)}{u-v-\frac{\eta}{2}} \\
& +\frac{1}{2 \pi i} \oint_{\mathcal{C}_{2}^{\prime}} d v \frac{\ln \left(\left(q(v) e^{-\beta \bar{\varepsilon}_{2}(v)}+(b(\beta)-1) e^{-\beta \bar{\varepsilon}_{3}(v)}\right) / b(\beta)\right)}{u-v+\frac{\eta}{2}}, \\
\ln \bar{\Lambda}_{2}^{(Q)}(u)= & \ln b(\beta)+\frac{1}{2 \pi i} \oint_{\mathcal{C}_{3}^{\prime}} d v \frac{\left.\ln \left(e^{-\beta \bar{\varepsilon}_{1}(v)}+(b(\beta)-1) e^{-\beta \bar{\varepsilon}_{4}(v)}\right) / b(\beta)\right)}{u-v-\frac{\eta}{2}} \\
& +\frac{1}{2 \pi i} \oint_{\mathcal{C}_{4}^{\prime}} d v \frac{\ln \left(\left(e^{-\beta \bar{\varepsilon}_{1}(v)}+(b(\beta)-1) e^{-\beta \bar{\varepsilon}_{4}(v)}\right) / b(\beta)\right)}{u-v+\frac{\eta}{2}},
\end{aligned}
$$

where the closed integral contour $\mathcal{C}_{1}^{\prime}$ (or $\mathcal{C}_{2}^{\prime}$ ) is surrounding the axis of $\operatorname{Im}(v)=\frac{1}{2}$ (or $-\frac{1}{2}$ ), while $\mathcal{C}_{3}^{\prime}$ (or $\mathcal{C}_{4}^{\prime}$ ) is surrounding the axis of $\operatorname{Im}(v)=1$ (or -1 ). With the help of the $t-W$ relations (5.34)-(5.35) and the integral representations (5.41)-(5.42), we can derive two NLIEs of the functions $\left\{\bar{\varepsilon}_{i}(u) \mid i=1, \ldots, 4\right\}$

$$
\begin{aligned}
& \ln \left(q(u) e^{-\beta \bar{\varepsilon}_{2}(u)}+(b(\beta)-1) e^{-\beta \bar{\varepsilon}_{3}(u)}\right)=\ln b(\beta) \\
& \quad+\frac{1}{2 \pi i} \oint_{\mathcal{C}_{1}^{\prime}} d v\left(\frac{1}{u-v}+\frac{1}{u-v-\eta}\right) \ln \left(\left(q(v) e^{-\beta \bar{\varepsilon}_{2}(v)}+(b(\beta)-1) e^{-\beta \bar{\varepsilon}_{3}(v)}\right) / b(\beta)\right) \\
& \quad+\frac{1}{2 \pi i} \oint_{\mathcal{C}_{2}^{\prime}} d v\left(\frac{1}{u-v+\eta}+\frac{1}{u-v}\right) \ln \left(\left(q(v) e^{-\beta \bar{\varepsilon}_{2}(v)}+(b(\beta)-1) e^{-\beta \bar{\varepsilon}_{3}(v)}\right) / b(\beta)\right), \\
& \ln \left(e^{-\beta \bar{\varepsilon}_{1}(u)}+(b(\beta)-1) e^{-\beta \bar{\varepsilon}_{4}(u)}\right)=\ln b(\beta) \\
& \quad+\frac{1}{2 \pi i} \oint_{\mathcal{C}_{3}^{\prime}} d v\left(\frac{1}{u-v}+\frac{1}{u-v-\eta}\right) \ln \left(\left(e^{-\beta \bar{\varepsilon}_{1}(v)}+(b(\beta)-1) e^{-\beta \bar{\varepsilon}_{4}(v)}\right) / b(\beta)\right) \\
& \quad+\frac{1}{2 \pi i} \oint_{\mathcal{C}_{4}^{\prime}} d v\left(\frac{1}{u-v+\eta}+\frac{1}{u-v}\right) \ln \left(\left(e^{-\beta \bar{\varepsilon}_{1}(v)}+(b(\beta)-1) e^{-\beta \bar{\varepsilon}_{4}(v)}\right) / b(\beta)\right) .
\end{aligned}
$$

It is believed that the analytic properties (5.40), the integral representations (5.41)-(5.42) and the NLIEs (5.43)-(5.44) might completely determine the functions $\left\{\bar{\varepsilon}_{i}(u) \mid=1, \ldots, 4\right\}$. Due to the fact that the roots and the poles of $\bar{\Lambda}_{1}^{(Q)}(u)$ locate nearly on the two lines with imaginary parts close to \pm 1 (see the decomposition (5.30)), we can use the Fourier transformation to obtain another integral representation of $\bar{\Lambda}_{1}^{(Q)}(u)$

$$
\begin{aligned}
\ln \bar{\Lambda}_{1}^{(Q)}(u) & =\int_{-\infty}^{+\infty} \frac{d v}{2 \cosh \pi(u-v)}\left\{\ln \bar{\Lambda}_{1}^{(Q)}\left(v+\frac{\eta}{2}\right)+\ln \bar{\Lambda}_{1}^{(Q)}\left(v-\frac{\eta}{2}\right)\right\} \\
& =\int_{-\infty}^{+\infty} \frac{d v}{2 \cosh \pi(u-v)}\left\{b(\beta) q(v) e^{-\beta \bar{\varepsilon}_{2}(v)}+\left(b^{2}(\beta)-b(\beta)\right) e^{-\beta \bar{\varepsilon}_{3}(v)}\right\} .
\end{aligned}
$$




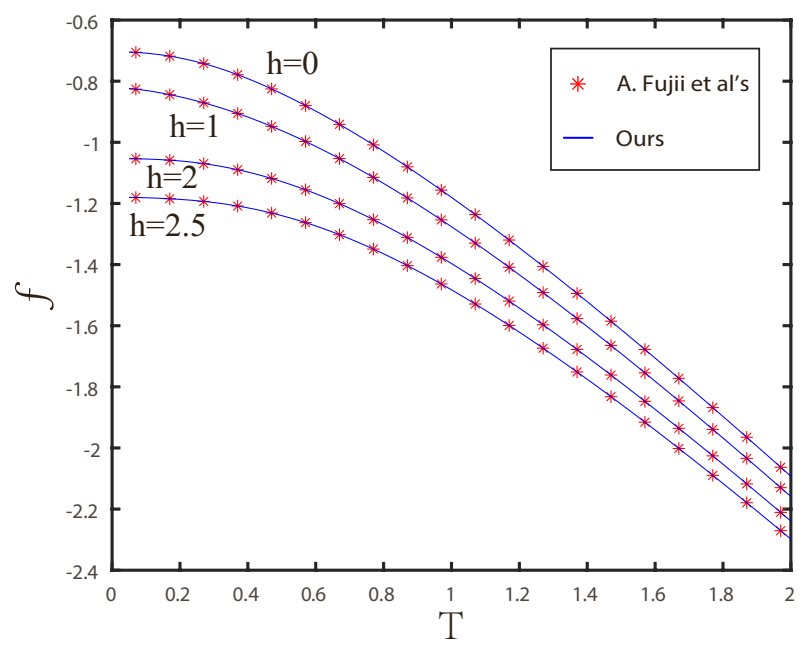

Figure 5. Free energy $f$ vs $T$ for the periodic SU(3) chain in different magnetic fields.

Finally we can obtain the free energy of the periodic SU(3) chain described by the Hamiltonian (5.13) with an external field $h$ as

$$
\begin{aligned}
f(\beta) & =2 J-\frac{1}{\beta} \ln \bar{\Lambda}_{1}^{(Q)}(0) \\
& =2 J-\frac{1}{\beta} \int_{-\infty}^{+\infty} \frac{d v}{2 \cosh \pi v} \ln \left(b(\beta) q(v) e^{-\beta \bar{\varepsilon}_{2}(v)}+\left(b^{2}(\beta)-b(\beta)\right) e^{-\beta \bar{\varepsilon}_{3}(v)}\right) .
\end{aligned}
$$

Using the numerical iterative procedure in appendix E, we obtain the free energy $f$ variation with temperature $T$ in different magnetic fields as shown in figure 5 . From this figure, we can conclude that our result coincides well that of [52] with a different approach. Moreover, we can obtain the HTE of the free energy of the SU(3)-invariant spin chain described by the Hamiltonian (5.13)-(5.14) (the details of the derivation is given in appendix F)

$$
f / T=-\ln \left(2 \cosh \left(\frac{h}{2 T}\right)+1\right)+\frac{J}{T} \frac{4}{2 \cosh \left(\frac{h}{2 T}\right)+1}-\frac{J^{2}}{T^{2}} \frac{24 \cosh \left(\frac{h}{2 T}\right)}{\left(2 \cosh \left(\frac{h}{2 T}\right)+1\right)^{2}}+\cdots
$$

\section{Conclusions}

In this paper, we have studied the thermodynamics of the Heisenberg chain at a finite temperature in anti-ferromagnetic regime via the recent developed $t-W$ method $[65,66]$. A novel nonlinear integral equation (4.2) which involves only one auxiliary function $\epsilon(u)$ has been given via the $t-W$ relation (3.3) satisfied by the associated transfer matrices. Together with some analytic property of the function $\epsilon(u)$ (see (3.26) and (4.4)), we solve the NLIE and obtain the free energy of the Heisenberg chain in different magnetic fields. Our results coincide well with those obtained by the other methods. Moreover, using the fusion technique we have obtained the $t-W$ relations (5.10)-(5.12) among the transfer matrices for the quantum integrable systems associated with $\mathrm{SU}(n)$, which allow one to derive the associated NLIEs. Taking the SU(3)-invariant quantum chain as an example, 
we construct the corresponding NLIEs which involve only four auxiliary functions. Solving the NLIEs, we obtain the free energy of the model.

We have proposed a more straightforward, efficient and easily extensible procedure to construct the associated NLIEs of obtaining free energy of the quantum spin chains. Our method can be easily generalized to the quantum integrable models solved by the offdiagonal Bethe ansatz method [73-75], which are associated with other Lie algebras such as $B_{n}, C_{n}$ and $D_{n}$. Moreover, for the $\mathrm{SU}(n)$-case, only $2 n-2$ functions have been involved in to obtain the eigenvalue $\Lambda^{(Q)}(u)$ of the original quantum transfer matrix.

\section{Acknowledgments}

The financial supports from the National Program for Basic Research of MOST (Grant Nos. 2016 YFA0300600 and 2016YFA0302104), National Natural Science Foundation of China (Grant Nos. 12074410, 12047502, 11934015, 11975183, 11947301 and 11774397), Major Basic Research Program of Natural Science of Shaanxi Province (Grant Nos. 2017KCT-12 and 2017ZDJC-32), Australian Research Council (Grant No. DP 190101529), the Strategic Priority Research Program of the Chinese Academy of Sciences (Grant No. XDB33000000), the fellowship of China Postdoctoral Science Foundation (Grant No. 2020M680724), and Double First-Class University Construction Project of Northwest University are gratefully acknowledged.

\section{A Proof of the $t-W$ relations (3.3) and (5.10)}

In this appendix we shall prove the operator relation (3.3) among the transfer matrices by using the fusion technique $[69,70]$ of the $R$-matrix.

For this purpose, let us introduce the (anti)symmetric subspaces $W^{( \pm)}$of $\mathbb{C}^{2} \otimes \mathbb{C}^{2}$ : $W^{( \pm)}=P_{12}^{( \pm)} \mathbb{C}^{2} \otimes \mathbb{C}^{2}$. Let $\{|i\rangle \mid i=1,2\}$ be an orthnormal basis of $\mathbb{C}^{2}$. It is easy to see that $W^{(+)}$is a 3 -dimensional subspace spanned by the orthnormal basis $\left\{|11\rangle, \frac{1}{\sqrt{2}}(|12\rangle+\right.$ $|21\rangle),|22\rangle\}$, while $W^{(-)}$is an 1-dimensional subspace spanned by $\left\{\frac{1}{\sqrt{2}}(|12\rangle-|21\rangle)\right\}$. The operator $\sigma_{1}^{z}+\sigma_{2}^{z}$ acts on $W^{( \pm)}$invariantly respectively. Denoted the action of $\sigma_{1}^{z}+\sigma_{2}^{z}$ on the subspace $W^{(+)}$by $\sigma_{\{12\}}^{z}$, it becomes a $3 \times 3$-matrix. In the basis $\left\{|11\rangle, \frac{1}{\sqrt{2}}(|12\rangle+|21\rangle),|22\rangle\right\}$, it reads

$$
\sigma_{\{12\}}^{z}=\left(\begin{array}{ccc}
2 & & \\
& 0 & \\
& & -2
\end{array}\right) .
$$

The QYBE (2.2) and the fusion condition (2.6) allow us to derive the relation

$$
R_{23}(u) R_{13}(u-\eta) P_{12}^{(-)}=P_{12}^{(-)} R_{23}(u) R_{13}(u-\eta) P_{12}^{(-)}=(u+\eta)(u-\eta) \times \text { id } .
$$

Direct calculation shows that

$$
P_{12}^{(+)} R_{23}(u) R_{13}(u-\eta) P_{12}^{(+)}=u R_{\{12\} 3}^{\left(1, \frac{1}{2}\right)}(u),
$$


where the fused $R$-matrix $R_{\{12\} 3}^{\left(1, \frac{1}{2}\right)}(u)$, in the basis $\left\{|11\rangle, \frac{1}{\sqrt{2}}(|12\rangle+|21\rangle),|22\rangle\right\}$, reads

$$
R_{\{12\} 3}^{\left(1, \frac{1}{2}\right)}(u)=\left(\begin{array}{cccccc}
u+\eta & & & & & \\
& u-\eta & \sqrt{2} & & & \\
& \sqrt{2} & u & & & \\
& & & u & \sqrt{2} & \\
& & & \sqrt{2} & u-\eta & \\
& & & & & u+\eta
\end{array}\right) .
$$

Keeping (3.1) in mind, let us introduce one-row monodromy matrix

$$
T_{0}(u)=R_{0 N}\left(u-\theta_{N}\right) \cdots R_{01}\left(u-\theta_{1}\right) .
$$

The relations (A.2) and (A.3) lead to

$$
\begin{aligned}
& P_{12}^{(-)} T_{2}(u) T_{1}(u-\eta) P_{12}^{(-)}=a(u) d(u-\eta) \times \mathrm{id}, \\
& P_{12}^{(+)} T_{2}(u) T_{1}(u-\eta) P_{12}^{(+)}=\prod_{l=1}^{N}\left(u-\theta_{l}\right) T_{\{12\}}^{\left(1, \frac{1}{2}\right)}(u),
\end{aligned}
$$

where the functions $a(u)$ and $d(u)$ are given by (3.4), and the fused monodromy matrix $T_{\{12\}}^{\left(1, \frac{1}{2}\right)}(u)$ can be expressed in terms of the fused $R$-matrix $R_{\{12\} 3}^{\left(1, \frac{1}{2}\right)}(u)$ given by (A.3)

$$
T_{\{12\}}^{\left(1, \frac{1}{2}\right)}(u)=R_{\{12\} N}^{\left(1, \frac{1}{2}\right)}\left(u-\theta_{N}\right) \cdots R_{\{12\} 1}^{\left(1, \frac{1}{2}\right)}\left(u-\theta_{1}\right) .
$$

Let us take the product of the transfer matrix $t(u)$ and $t(u-\eta)$ given by (3.1)

$$
\begin{aligned}
t(u) t(u-\eta)= & \operatorname{tr}_{12}\left\{e^{\frac{h \beta}{2}\left(\sigma_{1}^{z}+\sigma_{2}^{z}\right)} T_{2}(u) T_{1}(u-\eta)\right\} \\
= & \operatorname{tr}_{12}\left\{e^{\frac{h \beta}{2}\left(\sigma_{1}^{z}+\sigma_{2}^{z}\right)} T_{2}(u) T_{1}(u-\eta)\left(P_{12}^{(-)}+P_{12}^{(+)}\right)\right\} \\
= & \operatorname{tr}_{12}\left\{P_{12}^{(-)} e^{\frac{h \beta}{2}\left(\sigma_{1}^{z}+\sigma_{2}^{z}\right)} T_{2}(u) T_{1}(u-\eta) P_{12}^{(-)}\right\} \\
& \quad+\operatorname{tr}_{12}\left\{P_{12}^{(+)} e^{\frac{h \beta}{2}\left(\sigma_{1}^{z}+\sigma_{2}^{z}\right)} T_{2}(u) T_{1}(u-\eta) P_{12}^{(+)}\right\} \\
& \stackrel{(A .6)}{=} a(u) d(u-\eta) \times \mathrm{id} \\
\quad & \quad \operatorname{tr}_{12}\left\{P_{12}^{(+)} e^{\frac{h \beta}{2}\left(\sigma_{1}^{z}+\sigma_{2}^{z}\right)} P_{12}^{(+)} P_{12}^{(+)} T_{2}(u) T_{1}(u-\eta) P_{12}^{(+)}\right\} \\
& \stackrel{(A .7)}{=} a(u) d(u-\eta) \times \mathrm{id}+e^{\frac{h \beta}{2}} d(u) \operatorname{tr}_{12}\left\{P_{12}^{(+)} e^{\frac{h \beta}{2} \sigma_{\{12\}}^{z}} P_{12}^{(+)} T_{\{12\}}^{\left(1, \frac{1}{2}\right)}(u)\right\} .
\end{aligned}
$$

Then the fused transfer matrix $\mathbb{W}(u)$ is given by the tracing over the subspace $W^{(+)}$of the product of the fused monodromy matrix $T_{\{12\}}^{\left(1, \frac{1}{2}\right)}(u)$ and $e^{\frac{h \beta}{2} \sigma_{\{12\}}^{z}}$

$$
\mathbb{W}(u)=\operatorname{tr}_{12}\left\{e^{\frac{h \beta}{2} \sigma_{\{12\}}^{z}} T_{\{12\}}^{\left(1, \frac{1}{2}\right)}(u)\right\} .
$$

From the construction (A.4) and (A.8), we know that the matrix elements of the fused monodromy matrix $T_{\{12\}}^{\left(1, \frac{1}{2}\right)}(u)$, as a function of $u$, are operator-valued polynomials of degrees up to $N$. Finally we have completed the proof of the $t-W$ relation (3.3). 
Using the similar fusion procedure as that we have done for the $\mathrm{SU}(2)$ case, we can also derive the associated $t-W$ relations (5.10) among the fused transfer matrices $\left\{t_{i}^{(Q)}(u) \mid i=\right.$ $2, \cdots, n-1\}$ and their counterparts $\left\{\mathbb{W}_{i}^{(Q)}(u) \mid i=1, \cdots, n-1\right\}$.

\section{B Numerical scheme of the spin- $\frac{1}{2} \mathrm{XXX}$ closed chain}

For the convenience, let us introduce the function $\xi(u)$

$$
\xi(u)=\ln \left(\left(q(u)+\left(4 \cosh ^{2} \frac{h \beta}{2}-1\right) e^{-\beta \bar{\epsilon}(u)}\right) / 4 \cosh ^{2} \frac{h \beta}{2}\right) .
$$

Let us introduce two small positive parameters $\delta$ and $\Delta$ such that $0<\delta<\Delta<\frac{1}{2}$, and we can deform the integral contours in (4.2) without changing values of the resulting integrals as follows. The decomposition (the first identity of (3.23)) implies that the function $\xi(u)$ has singularities only on the straight $\operatorname{lines} \operatorname{Im}(u)= \pm \frac{1}{2}, \pm \frac{3}{2}$ and vanishes asymptotically, i.e., $\lim _{u \rightarrow \infty} \xi(u)=0$, which allows us to deform the integral contour $\mathcal{C}_{1}$ along the straight line $\operatorname{Im}(v)=\left(\frac{1}{2}-\delta\right)$ and the $\operatorname{line} \operatorname{Im}(v)=\left(\frac{1}{2}+\delta\right)$ (the contour $\mathcal{C}_{2}$ along the straight line $\operatorname{Im}(v)=-\left(\frac{1}{2}+\delta\right)$ and the $\operatorname{line} \operatorname{Im}(v)=-\left(\frac{1}{2}-\delta\right)$ anti-clockwise without changing the integral values in (4.2). Namely, we can have the integral representation

$$
\begin{aligned}
\ln \left(q(u)+\left(4 \cosh ^{2} \frac{h \beta}{2}-1\right) e^{-\beta \bar{\epsilon}(u)}\right)=2 \ln 2 \cosh \frac{h \beta}{2} \\
\quad-\frac{1}{2 \pi i} \int_{-\infty}^{+\infty} d v\left(\frac{1}{u-v-\left(\frac{1}{2}+\delta\right) \eta}+\frac{1}{u-v-\left(\frac{3}{2}+\delta\right) \eta}\right) \xi\left(v+\left(\frac{1}{2}+\delta\right) \eta\right) \\
\quad+\frac{1}{2 \pi i} \int_{-\infty}^{+\infty} d v\left(\frac{1}{u-v-\left(\frac{1}{2}-\delta\right) \eta}+\frac{1}{u-v-\left(\frac{3}{2}-\delta\right) \eta}\right) \xi\left(v+\left(\frac{1}{2}-\delta\right) \eta\right) \\
-\frac{1}{2 \pi i} \int_{-\infty}^{+\infty} d v\left(\frac{1}{u-v+\left(\frac{1}{2}-\delta\right) \eta}+\frac{1}{u-v+\left(\frac{3}{2}-\delta\right) \eta}\right) \xi\left(v-\left(\frac{1}{2}-\delta\right) \eta\right) \\
+\frac{1}{2 \pi i} \int_{-\infty}^{+\infty} d v\left(\frac{1}{u-v+\left(\frac{1}{2}+\delta\right) \eta}+\frac{1}{u-v+\left(\frac{3}{2}+\delta\right) \eta}\right) \xi\left(v-\left(\frac{1}{2}+\delta\right) \eta\right) .
\end{aligned}
$$

The above new integral representation allows us to compute the values of $\bar{\epsilon}\left(u \pm\left(\frac{1}{2}+\Delta\right) \eta\right)$ with $u \in \mathbb{R}$ provided that the values of $\xi(u)$ on the four straight lines $\operatorname{Im}(v)= \pm\left(\frac{1}{2}-\right.$ $\delta), \pm\left(\frac{1}{2}+\delta\right)$ are known. With the help of the analytical property (3.26) of the function $\bar{\epsilon}(u)$ and the Cauchy's theorem, we can compute the values $\bar{\epsilon}(u)$ on the four straight lines $\operatorname{Im}(u)= \pm\left(\frac{1}{2}-\delta\right), \pm\left(\frac{1}{2}+\delta\right)$ if we know its values on the two straight $\operatorname{lines} \operatorname{Im}(v)= \pm\left(\frac{1}{2}+\Delta\right)$. 
Namely, we have

$$
\begin{array}{ll}
\bar{\epsilon}\left(u+\left(\frac{1}{2}+\delta\right) \eta\right)=\frac{1}{2 \pi i} \int_{-\infty}^{+\infty} d v \frac{\bar{\epsilon}\left(v+\left(\frac{1}{2}+\Delta\right) \eta\right)}{u-v-(\Delta-\delta) \eta}-\frac{1}{2 \pi i} \int_{-\infty}^{+\infty} d v \frac{\bar{\epsilon}\left(v-\left(\frac{1}{2}+\Delta\right) \eta\right)}{u-v+(1+\Delta+\delta) \eta}, \quad u \in \mathbb{R}, \\
\bar{\epsilon}\left(u+\left(\frac{1}{2}-\delta\right) \eta\right)=\frac{1}{2 \pi i} \int_{-\infty}^{+\infty} d v \frac{\bar{\epsilon}\left(v+\left(\frac{1}{2}+\Delta\right) \eta\right)}{u-v-(\Delta+\delta) \eta}-\frac{1}{2 \pi i} \int_{-\infty}^{+\infty} d v \frac{\bar{\epsilon}\left(v-\left(\frac{1}{2}+\Delta\right) \eta\right)}{u-v+(1+\Delta-\delta) \eta}, \quad u \in \mathbb{R}, \\
\bar{\epsilon}\left(u-\left(\frac{1}{2}-\delta\right) \eta\right)=\frac{1}{2 \pi i} \int_{-\infty}^{+\infty} d v \frac{\bar{\epsilon}\left(v+\left(\frac{1}{2}+\Delta\right) \eta\right)}{u-v-(1+\Delta-\delta) \eta}-\frac{1}{2 \pi i} \int_{-\infty}^{+\infty} d v \frac{\bar{\epsilon}\left(v-\left(\frac{1}{2}+\Delta\right) \eta\right)}{u-v+(\Delta+\delta) \eta}, \quad u \in \mathbb{R}, \\
\bar{\epsilon}\left(u-\left(\frac{1}{2}+\delta\right) \eta\right)=\frac{1}{2 \pi i} \int_{-\infty}^{+\infty} d v \frac{\bar{\epsilon}\left(v+\left(\frac{1}{2}+\Delta\right) \eta\right)}{u-v-(1+\Delta+\delta) \eta}-\frac{1}{2 \pi i} \int_{-\infty}^{+\infty} d v \frac{\bar{\epsilon}\left(v-\left(\frac{1}{2}+\Delta\right) \eta\right)}{u-v+(\Delta-\delta) \eta}, \quad u \in \mathbb{R} .
\end{array}
$$

Now our numerical strategy can be constructed as follows. Starting from $\bar{\epsilon}_{(n)}\left(u \pm\left(\frac{1}{2}+\Delta\right) \eta\right)$, we can compute the values $\bar{\epsilon}_{(n)}\left(u \pm\left(\frac{1}{2}-\delta\right) \eta\right)$ and $\bar{\epsilon}_{(n)}\left(u \pm\left(\frac{1}{2}+\delta\right) \eta\right)$ with the help of the Cauchy's integrals (B.3)-(B.6). The integral representation (B.2) allows us to obtain $\bar{\epsilon}_{(n+1)}\left(u \pm\left(\frac{1}{2}+\Delta\right) \eta\right)$. Then repeat the above step again. Finally we might reach the solution of the integral equation (4.2) with the analytic property (3.26).

\section{High-temperature expansion of the Heisenberg chain}

For $\beta \rightarrow 0$, the function $\bar{w}(u)$ becomes independent of $u$ since the integrand in relation (4.2) has no poles in the area surrounded by the contours $\mathcal{C}_{1}$ and $\mathcal{C}_{2}$. Inserting $\bar{\epsilon}(u) \sim 0$ into the integral in relation (4.5) leads to the correct high-temperature entropy $-\beta f=\ln \Lambda(0) \sim$ $\ln 2$.

For small values of $\beta$, we seek $\bar{\epsilon}(u)$ as the series expansion

$$
\bar{\epsilon}(u)=\bar{\epsilon}_{1}(u)+\beta \bar{\epsilon}_{2}(u)+\cdots
$$

With regard to the expansion formula

$$
\begin{aligned}
\ln \left[q(u)+a e^{-\beta \bar{\epsilon}}\right]= & \ln (a+1)+\frac{\beta}{a+1}\left[\frac{2 J}{u^{2}+\frac{1}{4}}-a \bar{\epsilon}_{1}\right] \\
& +\frac{\beta^{2}}{2(a+1)^{2}}\left[a\left(\frac{2 J}{u^{2}+\frac{1}{4}}+\bar{\epsilon}_{1}\right)^{2}-2\left(a^{2}+a\right) \bar{\epsilon}_{2}\right]+\cdots,
\end{aligned}
$$

where we have set $a=4 \cosh ^{2}\left(\frac{h \beta}{2}\right)-1$ and the integral equation (4.2) transforms itself into an infinite sequence of coupled equations for the expansion functions $\left\{\bar{\epsilon}_{j}(u)\right\}$ :

$$
\begin{aligned}
a \bar{\epsilon}_{1}(u)= & \frac{2 J}{u^{2}+\frac{1}{4}}-\frac{1}{2 \pi i} \oint_{\mathcal{C}_{1}} d v\left(\frac{1}{u-v}+\frac{1}{u-v-i}\right)\left(\frac{2 J}{v^{2}+\frac{1}{4}}-a \bar{\epsilon}_{1}(v)\right) \\
& +\frac{1}{2 \pi i} \oint_{\mathcal{C}_{2}} d v\left(\frac{1}{u-v+i}+\frac{1}{u-v}\right)\left(\frac{2 J}{v^{2}+\frac{1}{4}}-a \bar{\epsilon}_{1}(v)\right) \\
2(a+1) \bar{\epsilon}_{2}(u)= & \left(\frac{2 J}{u^{2}+\frac{1}{4}}-\bar{\epsilon}_{1}(u)\right)^{2} \\
& -\frac{1}{2 \pi i} \oint_{\mathcal{C}_{1}} d v\left(\frac{1}{u-v}+\frac{1}{u-v-i}\right)\left(\left(\frac{2 J}{v^{2}+\frac{1}{4}}-\bar{\epsilon}_{1}(v)\right)^{2}-2(a+1) \bar{\epsilon}_{2}(v)\right) \\
& +\frac{1}{2 \pi i} \oint_{\mathcal{C}_{2}} d v\left(\frac{1}{u-v+i}+\frac{1}{u-v}\right)\left(\left(\frac{2 J}{v^{2}+\frac{1}{4}}-\bar{\epsilon}_{1}(v)\right)^{2}-2(a+1) \bar{\epsilon}_{2}(v)\right),
\end{aligned}
$$


etc. Note that the contour integrals of $\left\{\bar{\epsilon}_{j}(u)\right\}$ in r.h.s. vanishes because the function $\bar{\epsilon}(u)$ is analytic except some singularities on the axis $\operatorname{Im}(u)= \pm \frac{3}{2}$. Therefore, eq. (C.3) have two poles of $q(u)$ inside the contour $\mathcal{C}_{1}$ and $\mathcal{C}_{2}$ respectively. Using the residue theorem, we obtain the expression of $\bar{\epsilon}_{1}(u)$

$$
\bar{\epsilon}_{1}(u)=-\frac{1}{a} \frac{6 J}{u^{2}+\frac{9}{4}}
$$

Substituting $\bar{\epsilon}_{1}(u)$ into eq. (C.4), we have

$$
\bar{\epsilon}_{2}(u)=-\frac{1}{2\left(a^{2}+a\right)}\left(9\left(a-\frac{1}{a}\right)\left(\frac{2 J}{u^{2}+\frac{9}{4}}\right)^{2}+4(a-3) \frac{(2 J)^{2}}{u^{2}+\frac{9}{4}}\right) .
$$

The above expressions allow us to obtain the HTE (4.6) of the free energy.

\section{Bethe ansatz solution of the SU(3) model}

For the $\mathrm{SU}(3)$ model, it is well-known that eigenvalue of the quantum transfer matrix $t_{1}^{(Q)}(u)$ given by (5.16) can be obtained by the algebraic Bethe ansatz method [10], where $\Lambda_{1}^{(Q)}(u)$ is given in terms of a homogeneous $T-Q$ relation, namely,

$$
\begin{aligned}
\Lambda_{1}(u)= & {[(u-\eta \tau)(u+\eta \tau)]^{\frac{N}{2}} \frac{Q_{1}(u+\eta) Q_{2}(u-\eta)}{Q_{1}(u) Q_{2}(u)} } \\
& +[(u-\eta \tau)(u+\eta \tau-\eta)]^{\frac{N}{2}} \frac{Q_{2}(u+\eta)}{Q_{2}(u)} \\
& +[(u-\eta \tau+\eta)(u+\eta \tau)]^{\frac{N}{2}} \frac{Q_{1}(u-\eta)}{Q_{1}(u)}, \\
Q_{1}(u)= & \prod_{j=1}^{L_{1}}\left(u-\lambda_{j}^{(1)}\right), \quad Q_{2}(u)=\prod_{j=1}^{L_{2}}\left(u-\lambda_{j}^{(2)}\right) \quad L_{1}, L_{2}=0, \cdots, N .
\end{aligned}
$$

The two sets of parameters $\left\{\lambda_{j}^{(1)} \mid j=1, \cdots, L_{1} ; L_{1}=0, \cdots, N\right\}$ and $\left\{\lambda_{j}^{(2)} \mid j=1, \cdots, L_{2} ; L_{2}=\right.$ $0, \cdots, N\}$ satisfy the BAEs

$$
\begin{aligned}
& \left(\frac{\lambda_{i}^{(1)}-\eta \tau+\eta}{\lambda_{i}^{(1)}-\eta \tau}\right)^{\frac{N}{2}}=-\prod_{j=1}^{L_{2}} \frac{\lambda_{i}^{(1)}-\lambda_{j}^{(2)}-\eta}{\lambda_{i}^{(1)}-\lambda_{j}^{(2)}} \prod_{k=1}^{L_{1}} \frac{\lambda_{i}^{(1)}-\lambda_{k}^{(1)}+\eta}{\lambda_{i}^{(1)}-\lambda_{k}^{(1)}-\eta}, \quad i=1, \ldots, L_{1}, \\
& \left(\frac{\lambda_{l}^{(2)}+\eta \tau-\eta}{\lambda_{l}^{(1)}+\eta \tau}\right)^{\frac{N}{2}}=-\prod_{k=1}^{L_{1}} \frac{\lambda_{l}^{(2)}-\lambda_{k}^{(1)}+\eta}{\lambda_{l}^{(2)}-\lambda_{k}^{(1)}} \prod_{j=1}^{L_{2}} \frac{\lambda_{l}^{(2)}-\lambda_{j}^{(2)}-\eta}{\lambda_{l}^{(2)}-\lambda_{j}^{(2)}+\eta}, \quad l=1, \ldots, L_{2} .
\end{aligned}
$$

It was shown [34] that the eigenvalue $\Lambda_{1}^{(Q)}(u)$ corresponding to the state with $\left|\Lambda_{1}^{(Q)}(0)\right|_{\max }$ belongs to the sector of $L_{1}=L_{2}=\frac{N}{2}$ with all Bethe roots having the small imaginary part and $\lambda_{j}^{(2)}=\left(\lambda_{j}^{(1)}\right)^{*}$. Moreover $\operatorname{Im}\left(\lambda_{j}^{(i)}\right) \sim 0$ for a large $N$, the $T-Q$ relation (D.1) allows 
us to express $\bar{\Lambda}_{1}^{(Q)}(u)$ as

$$
\begin{aligned}
\bar{\Lambda}_{1}^{(Q)}(u)= & \frac{[(u-\eta \tau)(u+\eta \tau)]^{\frac{N}{2}}}{\prod_{j=1}^{\frac{N}{2}}\left(u-\lambda_{j}^{(1)}\right)\left(u-\lambda_{j}^{(2)}\right)} \frac{\prod_{j=1}^{\frac{N}{2}}\left(u-\lambda_{j}^{(2)}-\eta\right)\left(u-\lambda_{j}^{(1)}+\eta\right)}{[(u+\tau-\eta)(u-\tau+\eta)]^{\frac{N}{2}}} \\
& +\frac{(u-\eta \tau)^{\frac{N}{2}}}{\prod_{j=1}^{\frac{N}{2}}\left(u-\lambda_{j}^{(2)}\right)} \frac{\prod_{j=1}^{\frac{N}{2}}\left(u-\lambda_{j}^{(2)}+\eta\right)}{(u-\eta \tau+\eta)^{\frac{N}{2}}} \\
& +\frac{(u+\eta \tau)^{\frac{N}{2}}}{\prod_{j=1}^{\frac{N}{2}}\left(u-\lambda_{j}^{(1)}\right)} \frac{\prod_{j=1}^{\frac{N}{2}}\left(u-\lambda_{j}^{(1)}-\eta\right)}{(u+\eta \tau-\eta)^{\frac{N}{2}}} .
\end{aligned}
$$

For the fused quantum transfer matrix $t_{2}^{(Q)}(u)$, the corresponding eigenvalue $\Lambda_{2}^{(Q)}(u)$ can be expressed in terms of the $T-Q$ relation

$$
\begin{aligned}
\Lambda_{2}(u)= & {\left[\left(u-\eta \tau+\frac{3}{2} \eta\right)\left(u+\eta \tau-\frac{3}{2} \eta\right)\right]^{\frac{N}{2}} \frac{Q_{1}\left(u-\frac{\eta}{2}\right) Q_{2}\left(u+\frac{\eta}{2}\right)}{Q_{1}\left(u+\frac{\eta}{2}\right) Q_{2}\left(u-\frac{\eta}{2}\right)} } \\
& +\left[\left(u-\eta \tau+\frac{3}{2} \eta\right)\left(u+\eta \tau-\frac{\eta}{2}\right)\right]^{\frac{N}{2}} \frac{\left.Q_{2}\left(u-\frac{3}{2} \eta\right)\right)}{Q_{2}\left(u-\frac{\eta}{2}\right)} \\
& +\left[\left(u-\eta \tau+\frac{\eta}{2}\right)\left(u+\eta \tau-\frac{3}{2} \eta\right)\right]^{\frac{N}{2}} \frac{Q_{1}\left(u+\frac{3}{2} \eta\right)}{Q_{1}\left(u+\frac{\eta}{2}\right)},
\end{aligned}
$$

and the relation also allows us to express $\bar{\Lambda}_{2}^{(Q)}(u)$ as

$$
\begin{aligned}
\bar{\Lambda}_{2}^{(Q)}(u)= & \prod_{j=1}^{\frac{N}{2}} \frac{\left(u-\lambda_{j}^{(2)}+\frac{\eta}{2}\right)\left(u-\lambda_{j}^{(1)}-\frac{\eta}{2}\right)}{\left(u-\lambda_{j}^{(2)}-\frac{\eta}{2}\right)\left(u-\lambda_{j}^{(1)}+\frac{\eta}{2}\right)} \\
& +\frac{\left(u+\eta \tau-\frac{\eta}{2}\right)^{\frac{N}{2}}}{\prod_{j=1}^{\frac{N}{2}}\left(u-\lambda_{j}^{(2)}-\frac{\eta}{2}\right)} \frac{\prod_{j=1}^{\frac{N}{2}}\left(u-\lambda_{j}^{(2)}-\frac{3}{2} \eta\right)}{\left(u+\eta \tau-\frac{3}{2} \eta\right)^{\frac{N}{2}}} \\
& +\frac{\left(u-\eta \tau+\frac{\eta}{2}\right)^{\frac{N}{2}}}{\prod_{j=1}^{\frac{N}{2}}\left(u-\lambda_{j}^{(1)}+\frac{\eta}{2}\right)} \frac{\prod_{j=1}^{\frac{N}{2}}\left(u-\lambda_{j}^{(1)}+\frac{3}{2} \eta\right)}{\left(u-\eta \tau+\frac{3}{2} \eta\right)^{\frac{N}{2}}}
\end{aligned}
$$

\section{E Numerical scheme of the SU(3) model}

For the convenience, let us introduce the function $\xi_{1}(u)$ and $\xi_{2}(u)$

$$
\begin{aligned}
& \xi_{1}(u)=\ln \left(\left(q(u) e^{-\beta \bar{\varepsilon}_{2}(u)}+(b(\beta)-1) e^{-\beta \bar{\varepsilon}_{3}(u)}\right) / b(\beta)\right) \\
& \xi_{2}(u)=\ln \left(\left(e^{-\beta \bar{\varepsilon}_{1}(u)}+(b(\beta)-1) e^{-\beta \bar{\varepsilon}_{4}(u)}\right) / b(\beta)\right) .
\end{aligned}
$$

Similar as that we have done for the case of the XXX chain in appendix B, let us introduce two small positive parameters $\delta$ and $\Delta$ such that $0<\delta<\Delta<\frac{1}{2}$, and we can deform the integral contours in (5.43)-(5.44) without changing the values of resulting integrals as 
follows. The decomposition (the first identity of (5.34)) implies that the function $\xi_{1}(u)$ has singularities only on the straight $\operatorname{lines} \operatorname{Im}(u)= \pm \frac{1}{2}, \pm \frac{3}{2}$ and vanishes asymptotically, i.e., $\lim _{u \rightarrow \infty} \xi_{1}(u)=0$, which allow us to deform the integral contour $\mathcal{C}_{1}^{\prime}$ along the straight line $\operatorname{Im}(v)=\left(\frac{1}{2}-\delta\right)$ and the line $\operatorname{Im}(v)=\left(\frac{1}{2}+\delta\right)$ (the contour $\mathcal{C}_{2}^{\prime}$ along the straight $\operatorname{line} \operatorname{Im}(v)=$ $-\left(\frac{1}{2}+\delta\right)$ and the line $\left.\operatorname{Im}(v)=-\left(\frac{1}{2}-\delta\right)\right)$ anti-clockwise without changing the integral values in (5.43). In the same way, we can also deform the integral contour $\mathcal{C}_{3}^{\prime}$ along the straight line $\operatorname{Im}(v)=(1-\delta)$ and the line $\operatorname{Im}(v)=(1+\delta)$ (the contour $\mathcal{C}_{4}^{\prime}$ along the straight line $\operatorname{Im}(v)=-(1+\delta)$ and the line $\operatorname{Im}(v)=-(1-\delta))$ anti-clockwise without changing the integral values in (5.44). Namely, we have four integral representations as follows:

$$
\begin{aligned}
& \ln \left(q(u) e^{-\beta \bar{\varepsilon}_{2}(u)}+(b(\beta)-1) e^{-\beta \bar{\varepsilon}_{3}(u)}\right)=\ln b(\beta) \\
& -\frac{1}{2 \pi i} \int_{-\infty}^{+\infty} d v\left(\frac{1}{u-v-\left(\frac{1}{2}+\delta\right) \eta}+\frac{1}{u-v-\left(\frac{3}{2}+\delta\right) \eta}\right) \xi_{1}\left(v+\left(\frac{1}{2}+\delta\right) \eta\right) \\
& +\frac{1}{2 \pi i} \int_{-\infty}^{+\infty} d v\left(\frac{1}{u-v-\left(\frac{1}{2}-\delta\right) \eta}+\frac{1}{u-v-\left(\frac{3}{2}-\delta\right) \eta}\right) \xi_{1}\left(v+\left(\frac{1}{2}-\delta\right) \eta\right) \\
& -\frac{1}{2 \pi i} \int_{-\infty}^{+\infty} d v\left(\frac{1}{u-v+\left(\frac{1}{2}-\delta\right) \eta}+\frac{1}{u-v+\left(\frac{3}{2}-\delta\right) \eta}\right) \xi_{1}\left(v-\left(\frac{1}{2}-\delta\right) \eta\right) \\
& +\frac{1}{2 \pi i} \int_{-\infty}^{+\infty} d v\left(\frac{1}{u-v+\left(\frac{1}{2}+\delta\right) \eta}+\frac{1}{u-v+\left(\frac{3}{2}+\delta\right) \eta}\right) \xi_{1}\left(v-\left(\frac{1}{2}+\delta\right) \eta\right) \\
& \left.\bar{\varepsilon}_{1}(u)=\frac{1}{2 \pi i} \int_{-\infty}^{+\infty} d v\left(\frac{1}{u-v-(1+\delta) \eta} \xi_{1}\left(v+\left(\frac{1}{2}+\delta\right) \eta\right)-\frac{1}{u-v-(1-\delta) \eta}\right) \xi_{1}\left(v+\left(\frac{1}{2}-\delta\right) \eta\right)\right) \\
& \left.+\frac{1}{2 \pi i} \int_{-\infty}^{+\infty} d v\left(\frac{1}{u-v+(1-\delta) \eta} \xi_{1}\left(v-\left(\frac{1}{2}-\delta\right) \eta\right)-\frac{1}{u-v+(1+\delta) \eta}\right) \xi_{1}\left(v-\left(\frac{1}{2}+\delta\right) \eta\right)\right), \\
& \ln \left(e^{-\beta \bar{\varepsilon}_{1}(u)}+(b(\beta)-1) e^{-\beta \bar{\varepsilon}_{4}(u)}\right)=\ln b(\beta) \\
& -\frac{1}{2 \pi i} \int_{-\infty}^{+\infty} d v\left(\frac{1}{u-v-(1+\delta) \eta}+\frac{1}{u-v-(2+\delta) \eta}\right) \xi_{2}(v+(1+\delta) \eta) \\
& +\frac{1}{2 \pi i} \int_{-\infty}^{+\infty} d v\left(\frac{1}{u-v-(1-\delta) \eta}+\frac{1}{u-v-(2-\delta) \eta}\right) \xi_{2}(v+(1-\delta) \eta) \\
& -\frac{1}{2 \pi i} \int_{-\infty}^{+\infty} d v\left(\frac{1}{u-v+(1-\delta) \eta}+\frac{1}{u-v+(2-\delta) \eta}\right) \xi_{2}(v-(1-\delta) \eta) \\
& +\frac{1}{2 \pi i} \int_{-\infty}^{+\infty} d v\left(\frac{1}{u-v+(1+\delta) \eta}+\frac{1}{u-v+(2+\delta) \eta}\right) \xi_{2}(v-(1+\delta) \eta), \\
& \left.\bar{\varepsilon}_{2}(u)=\frac{1}{2 \pi i} \int_{-\infty}^{+\infty} d v\left(\frac{1}{u-v-\left(\frac{3}{2}+\delta\right) \eta} \xi_{2}(v+(1+\delta) \eta)-\frac{1}{u-v-\left(\frac{3}{2}-\delta\right) \eta}\right) \xi_{2}(v+(1-\delta) \eta)\right) \\
& \left.+\frac{1}{2 \pi i} \int_{-\infty}^{+\infty} d v\left(\frac{1}{u-v+\left(\frac{3}{2}-\delta\right) \eta} \xi_{2}(v-(1-\delta) \eta)-\frac{1}{u-v+\left(\frac{3}{2}+\delta\right) \eta}\right) \xi_{2}(v-(1+\delta) \eta)\right) .
\end{aligned}
$$

The above new integral representations allow us to compute the values of $\bar{\varepsilon}_{\{1,4\}}(u \pm(1+\Delta) \eta)$, $\bar{\varepsilon}_{\{2,3\}}\left(u \pm\left(\frac{1}{2}+\Delta\right) \eta\right), \bar{\varepsilon}_{1}(u \pm(1-\delta) \eta)$ and $\bar{\varepsilon}_{1}(u \pm(1+\delta) \eta)$ with $u \in \mathbb{R}$ provided that the values of $\xi_{1}(u)$ on the four straight $\operatorname{lines} \operatorname{Im}(v)= \pm\left(\frac{1}{2}-\delta\right), \pm\left(\frac{1}{2}+\delta\right)$ and the values of $\xi_{2}(u)$ 
on the other four straight lines $\operatorname{Im}(v)= \pm(1-\delta), \pm(1+\delta)$ are known. With the help of the analytical properties (5.40) of the function $\left\{\bar{\varepsilon}_{i}(u) \mid i=1, \ldots, 4\right\}$ and the Cauchy's theorem, we can compute the values $\bar{\varepsilon}_{\{2,3\}}(u)$ on the four straight lines $\operatorname{Im}(u)= \pm\left(\frac{1}{2}-\delta\right), \pm\left(\frac{1}{2}+\delta\right)$ and the values $\bar{\varepsilon}_{4}(u)$ on the other four straight lines $\operatorname{Im}(u)= \pm(1-\delta), \pm(1+\delta)$ if we know the values $\bar{\varepsilon}_{\{2,3\}}(u)$ on the two straight lines $\operatorname{Im}(v)= \pm\left(\frac{1}{2}+\Delta\right)$ and the values $\bar{\varepsilon}_{4}(u)$ on the two straight lines $\operatorname{Im}(v)= \pm(1+\Delta)$. Namely, we have

$$
\begin{aligned}
& \bar{\varepsilon}_{\{2,3\}}\left(u+\left(\frac{1}{2}+\delta\right) \eta\right)=\frac{1}{2 \pi i} \int_{-\infty}^{+\infty} d v \frac{\bar{\varepsilon}_{\{2,3\}}\left(v+\left(\frac{1}{2}+\Delta\right) \eta\right)}{u-v-(\Delta-\delta) \eta}-\frac{1}{2 \pi i} \int_{-\infty}^{+\infty} d v \frac{\bar{\varepsilon}_{\{2,3\}}\left(v-\left(\frac{1}{2}+\Delta\right) \eta\right)}{u-v+(1+\Delta+\delta) \eta}, \quad u \in \mathbb{R}, \\
& \bar{\varepsilon}_{\{2,3\}}\left(u+\left(\frac{1}{2}-\delta\right) \eta\right)=\frac{1}{2 \pi i} \int_{-\infty}^{+\infty} d v \frac{\bar{\varepsilon}_{\{2,3\}}\left(v+\left(\frac{1}{2}+\Delta\right) \eta\right)}{u-v-(\Delta+\delta) \eta}-\frac{1}{2 \pi i} \int_{-\infty}^{+\infty} d v \frac{\bar{\varepsilon}_{\{2,3\}}\left(v-\left(\frac{1}{2}+\Delta\right) \eta\right)}{u-v+(1+\Delta-\delta) \eta}, \quad u \in \mathbb{R}, \\
& \bar{\varepsilon}_{\{2,3\}}\left(u-\left(\frac{1}{2}-\delta\right) \eta\right)=\frac{1}{2 \pi i} \int_{-\infty}^{+\infty} d v \frac{\bar{\varepsilon}_{\{2,3\}}\left(v+\left(\frac{1}{2}+\Delta\right) \eta\right)}{u-v-(1+\Delta-\delta) \eta}-\frac{1}{2 \pi i} \int_{-\infty}^{+\infty} d v \frac{\bar{\varepsilon}_{\{2,3\}}\left(v-\left(\frac{1}{2}+\Delta\right) \eta\right)}{u-v+(\Delta+\delta) \eta}, \quad u \in \mathbb{R}, \\
& \bar{\varepsilon}_{\{2,3\}}\left(u-\left(\frac{1}{2}+\delta\right) \eta\right)=\frac{1}{2 \pi i} \int_{-\infty}^{+\infty} d v \frac{\bar{\varepsilon}_{\{2,3\}}\left(v+\left(\frac{1}{2}+\Delta\right) \eta\right)}{u-v-(1+\Delta+\delta) \eta}-\frac{1}{2 \pi i} \int_{-\infty}^{+\infty} d v \frac{\bar{\varepsilon}_{\{2,3\}}\left(v-\left(\frac{1}{2}+\Delta\right) \eta\right)}{u-v+(\Delta-\delta) \eta}, \quad u \in \mathbb{R}, \\
& \bar{\varepsilon}_{4}(u+(1+\delta) \eta)=\frac{1}{2 \pi i} \int_{-\infty}^{+\infty} d v \frac{\bar{\varepsilon}_{4}(v+(1+\Delta) \eta)}{u-v-(\Delta-\delta) \eta}-\frac{1}{2 \pi i} \int_{-\infty}^{+\infty} d v \frac{\bar{\varepsilon}_{4}(v-(1+\Delta) \eta)}{u-v+(2+\Delta+\delta) \eta}, \quad u \in \mathbb{R}, \\
& \bar{\varepsilon}_{4}(u+(1-\delta) \eta)=\frac{1}{2 \pi i} \int_{-\infty}^{+\infty} d v \frac{\bar{\varepsilon}_{4}(v+(1+\Delta) \eta)}{u-v-(\Delta+\delta) \eta}-\frac{1}{2 \pi i} \int_{-\infty}^{+\infty} d v \frac{\bar{\varepsilon}_{4}(v-(1+\Delta) \eta)}{u-v+(2+\Delta-\delta) \eta}, \quad u \in \mathbb{R}, \\
& \bar{\varepsilon}_{4}(u-(1-\delta) \eta)=\frac{1}{2 \pi i} \int_{-\infty}^{+\infty} d v \frac{\bar{\varepsilon}_{4}(v+(1+\Delta) \eta)}{u-v-(2+\Delta-\delta) \eta}-\frac{1}{2 \pi i} \int_{-\infty}^{+\infty} d v \frac{\bar{\varepsilon}_{4}(v-(1+\Delta) \eta)}{u-v+(\Delta+\delta) \eta}, \quad u \in \mathbb{R}, \\
& \bar{\varepsilon}_{4}(u-(1+\delta) \eta)=\frac{1}{2 \pi i} \int_{-\infty}^{+\infty} d v \frac{\bar{\varepsilon}_{4}(v+(1+\Delta) \eta)}{u-v-(2+\Delta+\delta) \eta}-\frac{1}{2 \pi i} \int_{-\infty}^{+\infty} d v \frac{\bar{\varepsilon}_{4}(v-(1+\Delta) \eta)}{u-v+(\Delta-\delta) \eta}, \quad u \in \mathbb{R} .
\end{aligned}
$$

Now our numerical strategy can be constructed as follows. Starting from $\bar{\varepsilon}_{\{1,4\}}^{(n)}(u \pm(1+\Delta) \eta)$, $\bar{\varepsilon}_{\{2,3\}}^{(n)}\left(u \pm\left(\frac{1}{2}+\Delta\right) \eta\right), \bar{\varepsilon}_{1}^{(n)}(u \pm(1-\delta) \eta)$ and $\bar{\varepsilon}_{1}^{(n)}(u \pm(1+\delta) \eta)$, we can compute the values $\bar{\varepsilon}_{\{2,3\}}^{(n)}\left(u \pm\left(\frac{1}{2}-\delta\right) \eta\right), \bar{\varepsilon}_{\{2,3\}}^{(n)}\left(u \pm\left(\frac{1}{2}+\delta\right) \eta\right), \bar{\varepsilon}_{4}^{(n)}(u \pm(1-\delta) \eta)$ and $\bar{\varepsilon}_{4}^{(n)}(u \pm(1+\delta) \eta)$ with the help of the Cauchy's integrals (E.7)-(E.14). The integral representation (B.2) allows us to obtain $\bar{\varepsilon}_{\{1,4\}}^{(n+1)}(u \pm(1+\Delta) \eta), \bar{\varepsilon}_{\{2,3\}}^{(n+1)}\left(u \pm\left(\frac{1}{2}+\Delta\right) \eta\right), \bar{\varepsilon}_{1}^{(n+1)}(u \pm(1-\delta) \eta)$ and $\bar{\varepsilon}_{1}^{(n+1)}(u \pm(1+\delta) \eta)$. Then repeat the above step again. Finally we might reach the solutions of the integral equations (5.43) and (5.44) with the analytic properties (5.40).

\section{F High-temperature expansion of the SU(3) model}

For $\beta \rightarrow 0$, the functions $\left\{\bar{\varepsilon}_{i}(u) \mid i=1, \ldots, 4\right\}$ become independent of $u$ since the integrand in relations (5.43) and (5.44) have no poles in the area surrounded by the contours $\left\{\mathcal{C}_{i}^{\prime} \mid i=\right.$ $1, \ldots, 4\}$. Inserting $\left\{\bar{\varepsilon}_{i}(u) \sim 0 \mid i=1, \ldots, 4\right\}$ into the integral in relation (5.45) leads to the correct high-temperature entropy $-\beta f=\ln \Lambda_{1}(0) \sim \ln 3$.

For small values of $\beta$, we seek $\left\{\bar{\varepsilon}_{i}(u) \mid i=1, \ldots, 4\right\}$ as the series expansion

$$
\bar{\varepsilon}_{i}(u)=\bar{\varepsilon}_{i 1}(u)+\beta \bar{\varepsilon}_{i 2}(u)+\cdots, \quad i=1, \ldots, 4 .
$$


With regard to the expansion formulas

$$
\begin{aligned}
\ln \left[q(u) e^{-\beta \bar{\varepsilon}_{2}}+(b-1) e^{-\beta \bar{\varepsilon}_{3}}\right]= & \ln b+\frac{\beta}{b}\left[\frac{2 J}{u^{2}+\frac{1}{4}}-\bar{\varepsilon}_{21}-(b-1) \bar{\varepsilon}_{31}\right] \\
+ & \frac{\beta^{2}}{2 b^{2}}\left[(b-1)\left(\frac{2 J}{u^{2}+\frac{1}{4}}-\bar{\varepsilon}_{21}+\bar{\varepsilon}_{31}\right)^{2}-2 b \bar{\varepsilon}_{22}-2\left(b^{2}-b\right) \bar{\varepsilon}_{32}\right]+\cdots, \\
\ln \left[e^{-\beta \bar{\varepsilon}_{1}}+(b-1) e^{\left.-\beta \bar{\varepsilon}_{4}\right]=}\right. & \ln b+\frac{\beta}{b}\left[-\bar{\varepsilon}_{11}-(b-1) \bar{\varepsilon}_{41}\right] \\
& +\frac{\beta^{2}}{2 b^{2}}\left[(b-1)\left(\bar{\varepsilon}_{11}-\bar{\varepsilon}_{41}\right)^{2}-2 b \bar{\varepsilon}_{12}-2\left(b^{2}-b\right) \bar{\varepsilon}_{42}\right]+\cdots,
\end{aligned}
$$

where we have set $b=2 \cosh \left(\frac{h \beta}{2}\right)+1$ and the integral equations (5.41)-(5.44) transforms theirself into an infinite sequence of coupled equations for the expansion functions $\left\{\bar{\varepsilon}_{i j}(u) \mid i=1, \ldots, 4\right\}$ :

$$
\begin{aligned}
& b \bar{\varepsilon}_{11}(u)=-\frac{1}{2 \pi i} \oint_{\mathcal{C}_{1}^{\prime}} d v \frac{1}{u-v-\frac{i}{2}}\left(\frac{2 J}{v^{2}+\frac{1}{4}}-\bar{\varepsilon}_{21}(v)-(b-1) \bar{\varepsilon}_{31}(v)\right) \\
& +\frac{1}{2 \pi i} \oint_{\mathcal{C}_{2}^{\prime}} d v \frac{1}{u-v+\frac{i}{2}}\left(\frac{2 J}{v^{2}+\frac{1}{4}}-\bar{\varepsilon}_{21}(v)-(b-1) \bar{\varepsilon}_{31}(v)\right), \\
& b \bar{\varepsilon}_{21}(u)=-\frac{1}{2 \pi i} \oint_{\mathcal{C}_{3}^{\prime}} d v \frac{1}{u-v-\frac{i}{2}}\left(-\bar{\varepsilon}_{11}(v)-(b-1) \bar{\varepsilon}_{41}(v)\right) \\
& +\frac{1}{2 \pi i} \oint_{\mathcal{C}_{4}^{\prime}} d v \frac{1}{u-v+\frac{i}{2}}\left(-\bar{\varepsilon}_{11}(v)-(b-1) \bar{\varepsilon}_{41}(v)\right), \\
& (b-1) \bar{\varepsilon}_{31}(u)=\frac{2 J}{u^{2}+\frac{1}{4}}-\bar{\varepsilon}_{21}(u) \\
& -\frac{1}{2 \pi i} \oint_{\mathcal{C}_{1}^{\prime}} d v\left(\frac{1}{u-v}+\frac{1}{u-v-i}\right)\left(\frac{2 J}{v^{2}+\frac{1}{4}}-\bar{\varepsilon}_{21}(v)-(b-1) \bar{\varepsilon}_{31}(v)\right) \\
& +\frac{1}{2 \pi i} \oint_{\mathcal{C}_{2}^{\prime}} d v\left(\frac{1}{u-v+i}+\frac{1}{u-v}\right)\left(\frac{2 J}{v^{2}+\frac{1}{4}}-\bar{\varepsilon}_{21}(v)-(b-1) \bar{\varepsilon}_{31}(v)\right), \\
& (b-1) \bar{\varepsilon}_{41}(u)=-\bar{\varepsilon}_{11}(u) \\
& -\frac{1}{2 \pi i} \oint_{\mathcal{C}_{3}^{\prime}} d v\left(\frac{1}{u-v}+\frac{1}{u-v-i}\right)\left(-\bar{\varepsilon}_{11}(v)-(b-1) \bar{\varepsilon}_{41}(v)\right) \\
& +\frac{1}{2 \pi i} \oint_{\mathcal{C}_{4}^{\prime}} d v\left(\frac{1}{u-v+i}+\frac{1}{u-v}\right)\left(-\bar{\varepsilon}_{11}(v)-(b-1) \bar{\varepsilon}_{41}(v)\right), \\
& 2 b^{2} \bar{\varepsilon}_{12}(u)=-\frac{1}{2 \pi i} \oint_{\mathcal{C}_{1}^{\prime}} d v \frac{1}{u-v-\frac{i}{2}}\left(\left(\frac{2 J}{v^{2}+\frac{1}{4}}-\bar{\varepsilon}_{21}(v)+\bar{\varepsilon}_{31}(v)\right)^{2}-2 b \bar{\varepsilon}_{22}(v)-2\left(b^{2}-b\right) \bar{\varepsilon}_{32}(v)\right) \\
& +\frac{1}{2 \pi i} \oint_{\mathcal{C}_{2}^{\prime}} d v \frac{1}{u-v+\frac{i}{2}}\left(\left(\frac{2 J}{v^{2}+\frac{1}{4}}-\bar{\varepsilon}_{21}(v)+\bar{\varepsilon}_{31}(v)\right)^{2}-2 b \bar{\varepsilon}_{22}(v)-2\left(b^{2}-b\right) \bar{\varepsilon}_{32}(v)\right), \\
& 2 b^{2} \bar{\varepsilon}_{22}(u)=-\frac{1}{2 \pi i} \oint_{\mathcal{C}_{3}^{\prime}} d v \frac{1}{u-v-\frac{i}{2}}\left(\left(\bar{\varepsilon}_{11}(v)-\bar{\varepsilon}_{41}(v)\right)^{2}-2 b \bar{\varepsilon}_{12}(v)-2\left(b^{2}-b\right) \bar{\varepsilon}_{42}(v)\right) \\
& +\frac{1}{2 \pi i} \oint_{\mathcal{C}_{4}^{\prime}} d v \frac{1}{u-v+\frac{i}{2}}\left(\left(\bar{\varepsilon}_{11}(v)-\bar{\varepsilon}_{41}(v)\right)^{2}-2 b \bar{\varepsilon}_{12}(v)-2\left(b^{2}-b\right) \bar{\varepsilon}_{42}(v)\right),
\end{aligned}
$$




$$
\begin{array}{r}
2\left(b^{2}-b\right) \bar{\varepsilon}_{32}(u)=(b-1)\left(\frac{2 J}{u^{2}+\frac{1}{4}}-\bar{\varepsilon}_{21}(u)+\bar{\varepsilon}_{31}(u)\right)^{2}-2 b \bar{\varepsilon}_{22}(u) \\
-\frac{1}{2 \pi i} \oint_{\mathcal{C}_{1}^{\prime}} d v\left(\frac{1}{u-v}+\frac{1}{u-v-i}\right)\left((b-1)\left(\frac{2 J}{v^{2}+\frac{1}{4}}-\bar{\varepsilon}_{21}(v)+\bar{\varepsilon}_{31}(v)\right)^{2}-2 b \bar{\varepsilon}_{22}(v)-2\left(b^{2}-b\right) \bar{\varepsilon}_{32}(v)\right) \\
+\frac{1}{2 \pi i} \oint_{\mathcal{C}_{2}^{\prime}} d v\left(\frac{1}{u-v+i}+\frac{1}{u-v}\right)\left((b-1)\left(\frac{2 J}{v^{2}+\frac{1}{4}}-\bar{\varepsilon}_{21}(v)+\bar{\varepsilon}_{31}(v)\right)^{2}-2 b \bar{\varepsilon}_{22}(v)-2\left(b^{2}-b\right) \bar{\varepsilon}_{32}(v)\right), \\
2\left(b^{2}-b\right) \bar{\varepsilon}_{42}(u)=(b-1)\left(\bar{\varepsilon}_{11}(u)-\bar{\varepsilon}_{41}(u)\right)^{2}-2 b \bar{\varepsilon}_{12}(u) \\
-\frac{1}{2 \pi i} \oint_{\mathcal{C}_{3}^{\prime}} d v\left(\frac{1}{u-v}+\frac{1}{u-v-i}\right)\left((b-1)\left(\bar{\varepsilon}_{11}(v)-\bar{\varepsilon}_{41}(v)\right)^{2}-2 b \bar{\varepsilon}_{12}(v)-2\left(b^{2}-b\right) \bar{\varepsilon}_{42}(v)\right) \\
+\frac{1}{2 \pi i} \oint_{\mathcal{C}_{4}^{\prime}} d v\left(\frac{1}{u-v+i}+\frac{1}{u-v}\right)\left((b-1)\left(\bar{\varepsilon}_{11}(v)-\bar{\varepsilon}_{41}(v)\right)^{2}-2 b \bar{\varepsilon}_{12}(v)-2\left(b^{2}-b\right) \bar{\varepsilon}_{42}(v)\right) .
\end{array}
$$

Note that the contour integrals of $\left\{\bar{\varepsilon}_{i j}(u) \mid i=2, \ldots, 4\right\}$ in r.h.s. vanishes because the analytic properties (5.40) of the functions $\left\{\bar{\varepsilon}_{i}(u) \mid i=2, \ldots, 4\right\}$. Therefore, eq. (F.4) have two poles of $q(u)$ inside the contour $\mathcal{C}_{1}^{\prime}$ and $\mathcal{C}_{2}^{\prime}$ respectively. Using the residue theorem, we obtain the expression of $\bar{\varepsilon}_{11}(u)$

$$
\bar{\varepsilon}_{11}(u)=-\frac{1}{b} \frac{4 J}{u^{2}+1} .
$$

Substituting $\bar{\varepsilon}_{11}(u)$ into eq. (F.5) and applying once again the residue theorem, we have

$$
\bar{\varepsilon}_{21}(u)=-\frac{1}{b} \frac{6 J}{u^{2}+\frac{9}{4}} .
$$

Through the similar processes, we can obtain the rest of the expressions $\left\{\bar{\varepsilon}_{i j}(u) \mid i=2, \ldots, 4\right\}$

$$
\begin{aligned}
& \bar{\varepsilon}_{31}(u)=-\frac{1}{b} \frac{6 J}{u^{2}+\frac{9}{4}}, \\
& \bar{\varepsilon}_{41}(u)=-\frac{1}{b(b-1)} \frac{8 J}{u^{2}+4}, \\
& \bar{\varepsilon}_{12}(u)=-\frac{b-1}{2 b^{2}}\left(\frac{4}{\left(u^{2}+1\right)^{2}}+\frac{2}{u^{2}+1}\right)(2 J)^{2}, \\
& \bar{\varepsilon}_{22}(u)=-\frac{1}{2 b^{4}}\left(\frac{9\left(b^{2}-1\right)}{\left(u^{2}+\frac{9}{4}\right)^{2}}+\frac{\left(4 b^{2}-3 b-9\right)}{u^{2}+\frac{9}{4}}\right)(2 J)^{2}, \\
& \bar{\varepsilon}_{32}(u)=-\frac{1}{2 b^{4}}\left(\frac{9\left(b^{3}-b-1\right)}{\left(u^{2}+\frac{9}{4}\right)^{2}}+\frac{\left(4 b^{4}-4 b^{3}-4 b^{2}+3 b+9\right)}{(b-1)\left(u^{2}+\frac{9}{4}\right)}\right)(2 J)^{2}, \\
& \bar{\varepsilon}_{42}(u)=-\frac{1}{2\left(b^{3}-b^{2}\right)}\left(\frac{16\left(b^{2}-b-1\right)}{(b-1)\left(u^{2}+4\right)^{2}}+\frac{2\left(3 b^{2}-2 b-9\right)}{b\left(u^{2}+4\right)}\right)(2 J)^{2} .
\end{aligned}
$$

Substituting the above results into the free energy eq. (5.45) and integral, we have the HTE of the free energy

$$
f / T=-\ln \left(2 \cosh \left(\frac{h}{2 T}\right)+1\right)+\frac{J}{T} \frac{4}{2 \cosh \left(\frac{h}{2 T}\right)+1}-\frac{J^{2}}{T^{2}} \frac{24 \cosh \left(\frac{h}{2 T}\right)}{\left(2 \cosh \left(\frac{h}{2 T}\right)+1\right)^{2}}+\cdots
$$

Finally we have completed the proof of (5.46). 
Open Access. This article is distributed under the terms of the Creative Commons Attribution License (CC-BY 4.0), which permits any use, distribution and reproduction in any medium, provided the original author(s) and source are credited.

\section{References}

[1] R.J. Baxter, Exactly Solved Models in Statistical Mechanics, Academic Press (1982).

[2] J.M. Maldacena, The large $N$ limit of superconformal field theories and supergravity, Adv. Theor. Math. Phys. 2 (1998) 231 [hep-th/9711200] [InSPIRE].

[3] N. Beisert et al., Review of AdS/CFT integrability: an overview, Lett. Math. Phys. 99 (2012) 1.

[4] J. de Gier and F.H.L. Essler, Bethe Ansatz Solution of the Asymmetric Exclusion Process with Open Boundaries, Phys. Rev. Lett. 95 (2005) 240601 [cond-mat/0508707] [INSPIRE].

[5] J. Sirker, R.G. Pereira and I. Affleck, Diffusion and ballistic transport in one-dimensional quantum systems, Phys. Rev. Lett. 103 (2009) 216602.

[6] J. Dukelsky, S. Pittel and G. Sierra, Colloquium: Exactly solvable Richardson-Gaudin models for many-body quantum systems, Rev. Mod. Phys. 76 (2004) 643 [nucl-th/0405011] [INSPIRE].

[7] X.-W. Guan, M.T. Batchelor and C. Lee, Fermi gases in one dimension: From bethe ansatz to experiments, Rev. Mod. Phys. 85 (2013) 1633.

[8] H. Bethe, On the theory of metals. 1. Eigenvalues and eigenfunctions for the linear atomic chain, Z. Phys. 71 (1931) 205 [INSPIRE].

[9] L.D. Faddeev, E.K. Sklyanin and L.A. Takhtajan, The Quantum Inverse Problem Method. 1, Theor. Math. Phys. 40 (1980) 688 [Teor. Mat. Fiz. 40 (1979) 194] [INSPIRE].

[10] V.E. Korepin, N.M. Bogoliubov and A.G. Izergin, Quantum Inverse Scattering Method and Correlation Function, Cambridge University Press, (1993).

[11] N.Y. Reshetikhin, A method of functional equations in the theory of exactly solvable quantum systems, Sov. Phys. JETP 57 (1983) 691.

[12] E.K. Sklyanin, Separation of variables in the Gaudin model, J. Sov. Math. 47 (1989) 2473 [Zap. Nauchn. Semin. 164 (1987) 151] [INSPIRE].

[13] E.K. Sklyanin, Separation of variables - new trends, Prog. Theor. Phys. Suppl. 118 (1995) 35 [solv-int/9504001] [INSPIRE].

[14] Y. Wang, W. -L. Yang, J. Cao and K. Shi, Off-Diagonal Bethe Ansatz for Exactly Solvable Models, Springer Press (2015).

[15] P. Baseilhac and S. Belliard, The half-infinite XXZ chain in Onsager's approach, Nucl. Phys. $B 873$ (2013) 550 [arXiv:1211.6304] [INSPIRE].

[16] J. Avan, S. Belliard, N. Grosjean and R.A. Pimenta, Modified algebraic Bethe ansatz for XXZ chain on the segment - III - Proof, Nucl. Phys. B 899 (2015) 229 [arXiv:1506.02147] [INSPIRE].

[17] C.-N. Yang and C.P. Yang, Thermodynamics of one-dimensional system of bosons with repulsive delta function interaction, J. Math. Phys. 10 (1969) 1115 [INSPIRE]. 
[18] C.P. Yang, One-dimensional system of bosons with repulsive $\delta$-function interactions at a finite TemperatureT, Phys. Rev. A 2 (1970) 154.

[19] E.H. Lieb and W. Liniger, Exact analysis of an interacting Bose gas. 1. The general solution and the ground state, Phys. Rev. 130 (1963) 1605 [INSPIRE].

[20] E.H. Lieb, Exact Analysis of an Interacting Bose Gas. 2. The Excitation Spectrum, Phys. Rev. 130 (1963) 1616 [INSPIRE].

[21] M. Gaudin, Thermodynamics of the Heisenberg Ising ring for $\Delta \gtrsim 1$, Phys. Rev. Lett. 26 (1971) 1301 [INSPIRE].

[22] M. Takahashi, Prog. Theor. Phys. 46 (1971) 401.

[23] M. Takahashi, Thermodynamics of one-dimensional solvable models, Cambridge University Press (2005).

[24] H. Johannesson, The integrable SU(n) heisenberg model at finite temperature, Phys. Lett. A 116 (1986) 133.

[25] P. Schlottmann, Logarithmic singularities in the susceptibility of the antiferromagnetic $S U(n)$ heisenberg model, Phys. Rev. B 45 (1992) 5293.

[26] K. Lee, Critical behavior of the $\mathrm{SU}(N)$-invariant Heisenberg ferromagnet in one dimension, J. Korean Phys. Soc. 27 (1994) 205.

[27] K. Lee, Low-temperature specific heat of the generalized antiferromagnetic SU(N) Heisenberg model with and without a field, Phys. Lett. A 187 (1994) 112.

[28] M. Suzuki, Transfer-matrix method and monte carlo simulation in quantum spin systems, Phys. Rev. B 31 (1985) 2957.

[29] T. Koma, Thermal bethe-ansatz method for the one-dimensional heisenberg model, Prog. Theor. Phys. 78 (1987) 1213.

[30] J. Suzuki, Y. Akutsu and M. Wadati, A new approach to quantum spin chains at finite temperature, J. Phys. Soc. Jap. 59 (1990) 2667.

[31] A. Klümper, Free energy and correlation lengths of quantum chains related to restricted solid-on-solid lattice models, Annals Phys. 1 (1992) 540.

[32] A. Klümper, The spin-1/2 Heisenberg chain: thermodynamics, quantum criticality and spin-Peierls exponents, Eur. Phys. J. B 5 (1998) 677.

[33] C. Destri and H.J. de Vega, New thermodynamic Bethe ansatz equations without strings, Phys. Rev. Lett. 69 (1992) 2313 [INSPIRE].

[34] F.H. Essler, H. Frahm, F. Göhmann, A. Klümper and V.E. Korepin, The one-dimensional Hubbard model, Cambridge University Press (2005).

[35] A.N. Kirillov and N.Y. Reshetikhin, Exact solution of the integrable XXZ Heisenberg model with arbitrary spin. I. The ground state and the excitation spectrum, J. Phys. A 20 (1987) 1565 [INSPIRE].

[36] V. Bazhanov and N. Reshetikhin, Restricted Solid on Solid Models Connected With Simply Based Algebras and Conformal Field Theory, J. Phys. A 23 (1990) 1477 [InSPIRE].

[37] A. Klumper and P.A. Pearce, Conformal weights of RSOS lattice models and their fusion hierarchies, Physica A 183 (1992) 304 [INSPIRE]. 
[38] A. Kuniba, T. Nakanishi and J. Suzuki, Functional relations in solvable lattice models. 1: Functional relations and representation theory, Int. J. Mod. Phys. A 9 (1994) 5215 [hep-th/9309137] [INSPIRE].

[39] Z. Tsuboi, Analytic Bethe ansatz and functional equations for Lie superalgebra $s l(r+1 \mid s+1)$, J. Phys. A 30 (1997) 7975 [arXiv:0911.5386] [INSPIRE].

[40] Z. Tsuboi, Analytic Bethe Ansatz And Functional Equations Associated With Any Simple Root Systems Of The Lie Superalgebra sl $(r+1 \mid s+1)$, Physica A 252 (1998) 565 [arXiv:0911.5387] [INSPIRE].

[41] G. Juttner, A. Klumper and J. Suzuki, From fusion hierarchy to excited state TBA, Nucl. Phys. B 512 (1998) 581 [hep-th/9707074] [INSPIRE].

[42] A. Klumper and M.T. Batchelor, An analytic treatment of finite-size corrections in the spin-1 antiferromagnetic XXZ chain, J. Phys. A 23 (1990) L189 [InSPIRE].

[43] A. Klumper, M.T. Batchelor and P.A. Pearce, Central charges of the 6-and 19-vertex models with twisted boundary conditions, J. Phys. A 24 (1991) 3111 [InSPIRE].

[44] A. Klumper, T. Wehner and J. Zittartz, Conformal spectrum of the six-vertex model, J. Phys. A 26 (1993) 2815.

[45] J. Benz, T. Fukui, A. Klümper and C. Scheeren, On the finite temperature drude weight of the anisotropic heisenberg chain, J. Phys. Soc. Jap. 74 (2005) 181.

[46] J. Suzuki, Spinons in magnetic chains of arbitrary spins at finite temperature, J. Phys. A 32 (1999) 2341 [cond-mat/9807076] [INSPIRE].

[47] G. Jüttner, A. Klümper and J. Suzuki, Exact thermodynamics and Luttinger liquid properties of the integrable t-J model, Nucl. Phys. B 487 (1997) 650 [cond-mat/9611058] [InSPIRE].

[48] A. Klümper and A.A. Zvyagin, Exact thermodynamics of disordered impurities in quantum spin chains, Phys. Rev. Lett. 81 (1998) 4975.

[49] A. Klümper, T. Wehner and J. Zittartz, Thermodynamics of the quantum Perk-Schultz model, J. Phys. A 30 (1997) 1897.

[50] G.A.P. Ribeiro and A. Klumper, Thermodynamics of antiferromagnetic alternating spin chains, Nucl. Phys. B 801 (2008) 247 [arXiv:0712.3765] [InSPIRE].

[51] J. Suzuki, Quantum Jacobi-Trudi formula and $E_{8}$ structure in the Ising model in a field, Nucl. Phys. B 528 (1998) 683 [cond-mat/9805241] [INSPIRE].

[52] A. Fujii and A. Klümper, Anti-symmetrically fused model and non-linear integral equations in the three-state Uimin-Sutherland model, Nucl. Phys. B 546 (1999) 751.

[53] M. Takahashi, Simplification of thermodynamic Bethe-ansatz equations, in Physics and Combinatrics, eds. A.K. Kirillov and N. Liskova, World Scientific, Singapore (2001), pp. 299-304, [cond-mat/0010486].

[54] T. Nishino, Density matrix renormalization group method for $2 d$ classical models, J. Phys. Soc. Jap. 64 (1995) 3598.

[55] Z. Tsuboi, Nonlinear integral equations for thermodynamics of the $S L(r+1)$ Uimin-Sutherland model, J. Phys. A 36 (2003) 1493 [cond-mat/0212280] [InSPIRE].

[56] M. Shiroishi and M. Takahashi, Integral Equation Generates High-Temperature Expansion of the Heisenberg Chain, Phys. Rev. Lett. 89 (2002) 117201 [cond-mat/0205180] [InSPIRE]. 
[57] Y.-K. Huang, P. Chen and Y.-J. Kao, Accurate computation of low-temperature thermodynamics for quantum spin chains, Phys. Rev. B 86 (2012) 235102.

[58] R.J. Bursill, T. Xiang and G.A. Gehring, The density matrix renormalization group for a quantum spin chain at non-zero temperature, J. Phys. Condens. Matter 8 (1996) L583.

[59] X. Wang and T. Xiang, Transfer-matrix density-matrix renormalization-group theory for thermodynamics of one-dimensional quantum systems, Phys. Rev. B 56 (1997) 5061.

[60] T. Xiang, Thermodynamics of quantum Heisenberg spin chains, Phys. Rev. B 58 (1998) 9142 [cond-mat/9808179] [INSPIRE].

[61] F. Naef, X. Wang, X. Zotos and W. von der Linden, Autocorrelations from the transfer-matrix density-matrix renormalization-group method, Phys. Rev. B 60 (1999) 359.

[62] J. Sirker, Thermodynamics of a one-dimensionalS=12spin-orbital model, Phys. Rev. B 69 (2004) 104428.

[63] H.T. Lu, Y.J. Wang, S. Qin and T. Xiang, Zigzag spin chains with antiferromagnetic-ferromagnetic interactions: Transfer-matrix renormalization group study, Phys. Rev. B 74 (2006) 134425.

[64] S. Sota and T. Tohyama, Low-temperature density matrix renormalization group study on spin-1/2 frustrated spin chains, J. Phys. Conf. Ser. 200 (2010) 012191.

[65] Y. Qiao, P. Sun, J. Cao, W.-L. Yang, K. Shi and Y. Wang, Exact ground state and elementary excitations of a topological spin chain, Phys. Rev. B 102 (2020) 085115 [arXiv: 2003.07089] [INSPIRE].

[66] Y. Qiao, J. Cao, W.-L. Yang, K. Shi and Y. Wang, Exact surface energy and helical spinons in the XXZ spin chain with arbitrary nondiagonal boundary fields, Phys. Rev. B 103 (2021) 220401 [arXiv: 2102.02643] [INSPIRE].

[67] B. Sutherland, A General Model for Multicomponent Quantum Systems, Phys. Rev. B 12 (1975) 3795 [INSPIRE].

[68] J.H. Perk and C.L. Schultz, New families of commuting transfer matrices in q-state vertex models, Phys. Lett. A $8 \mathbf{4}$ (1981) 407.

[69] P.P. Kulish and E.K. Sklyanin, Quantum spectral transform method. Recent developments, Lect. Notes Phys. 151 (1982) 61 [INSPIRE].

[70] A.N. Kirillov and N.Y. Reshetikhin, Exact solution of the heisenberg XXZ model of spin s, J. Sov. Math. 35 (1986) 2627.

[71] J. Cao, W.-L. Yang, K. Shi and Y. Wang, Nested off-diagonal Bethe ansatz and exact solutions of the $\mathrm{SU}(N)$ spin chain with generic integrable boundaries, JHEP 04 (2014) 143 [arXiv: 1312.4770] [INSPIRE].

[72] J. Damerau and A. Klümper, Nonlinear integral equations for the thermodynamics of thesl(4)-symmetric uimin-sutherland model, J. Stat. Mech. 2006 (2006) P12014.

[73] G.-L. Li et al., Exact solution of the sp(4) integrable spin chain with generic boundaries, JHEP 05 (2019) 067 [arXiv:1812.03618] [INSPIRE].

[74] G.-L. Li et al., Off-diagonal bethe ansatz on the so(5) spin chain, Nucl. Phys. B 946 (2019) 114719 .

[75] G.-L. Li et al., Off-diagonal Bethe Ansatz for the $D_{3}^{(1)}$ model, JHEP 12 (2019) 051 [arXiv: 1909.08534] [INSPIRE]. 